\title{
Passive tracer in a slowly decorrelating random flow with a large
}

\section{mean}

\author{
Tomasz Komorowski * $\quad$ Lenya Ryzhik ${ }^{\dagger}$
}

October 1, 2018

\begin{abstract}
We consider the movement of a particle advected by a random flow of the form $\mathbf{v}+\delta \mathbf{F}(\mathbf{x})$, with $\mathbf{v} \in \mathbb{R}^{d}$ a constant drift, $\mathbf{F}(\mathbf{x})$ - the fluctuation - given by a zero mean, stationary random field and $\delta \ll 1$ so that the drift dominates over the fluctuation. The two-point correlation matrix $\mathbf{R}(\mathbf{x})$ of the random field decays as $|\mathbf{x}|^{2 \alpha-2}$, as $|\mathbf{x}| \rightarrow+\infty$ with $\alpha<1$. The Kubo formula for the effective diffusion coefficient obtained in 17 for rapidly decorrelating fields diverges when $1 / 2 \leq \alpha<1$. We show formally that on the time scale $\delta^{-1 / \alpha}$ the deviation of the trajectory from its mean $\mathbf{y}(t)=\mathbf{x}(t)-\mathbf{v} t$ converges to a fractional Brownian motion $B_{\alpha}(t)$ in this range of the exponent $\alpha$. We also prove rigorously upper and lower bounds which show that $\mathbb{E}\left[|\mathbf{y}(t)|^{2}\right]$ converges to zero for times $t \ll \delta^{-1 / \alpha}$ and to infinity on time scales $t \gg \delta^{-1 / \alpha}$ as $\delta \rightarrow 0$ when $\alpha \in(1 / 2,1)$. On the other hand, when $\alpha<1 / 2$ non-trivial behavior is observed on the time-scale $O\left(\delta^{-2}\right)$.
\end{abstract}

\section{Introduction}

The position of a tracer in a random flow is described by the ordinary differential equation

$$
\frac{d \mathbf{X}(t ; \mathbf{x})}{d t}=\mathbf{V}(t, \mathbf{X}(t ; \mathbf{x})), \quad \mathbf{X}(0 ; \mathbf{x})=\mathbf{x}
$$

where $\mathbf{V}(t, \mathbf{x})$ is a random field. This random model is frequently used to describe the motion of a particle in a turbulent flow of fluid, where $\mathbf{V}(t, \mathbf{x})$ is the Eulerian velocity field. The long-time behavior of solutions of (1.1) has been extensively studied, especially when $\mathbf{V}(t, \mathbf{x})$ is sufficiently rapidly mixing in time and space - see [21 for an extensive overview. Roughly speaking, one expects that the trajectory $\mathbf{X}(t ; \mathbf{x})$ behaves diffusively if the velocity field decorrelates sufficiently fast in time and space while an anomalous behavior is observed if $\mathbf{V}(t, \mathbf{x})$ has long range correlations. This problem remains largely open when $\mathbf{V}(\mathbf{x})$ is both time-independent and of zero mean, as then there is no mechanism to "move the particle around" and employ the decorrelating properties of the random field. Therefore, to simplify matters, we assume that the flow satisfies the Taylor hypothesis, that is, it has a non-zero mean drift which dominates over the amplitude of its fluctuations. This means that we can write $\mathbf{V}(\mathbf{x})=\mathbf{v}+\delta \mathbf{F}(\mathbf{x})$, where $\mathbf{v} \neq \mathbf{0}$ is a constant vector with, say, $|\mathbf{v}|=1$, and the parameter $\delta \ll 1$. The fluctuation $\mathbf{F}(\mathbf{x})$ is spatially homogeneous, of zero mean and is

\footnotetext{
*Institute of Mathematics, UMCS, pl. Marii Curie-Skłodowskiej 1, Lublin 20-031, Poland; komorow@hektor.umcs. lublin.pl

${ }^{\dagger}$ Department of Mathematics, University of Chicago, Chicago, IL 60637, USA; ryzhik@math.uchicago.edu
} 
divergence-free: $\nabla \cdot \mathbf{F}(\mathbf{x})=0$. It has been shown in [17] that if the field $\mathbf{F}(\mathbf{x})$ is sufficiently strongly mixing in space then the scaled trajectories

$$
\mathbf{y}\left(t / \delta^{2} ; \mathbf{x}\right):=\mathbf{x}\left(t / \delta^{2} ; \mathbf{x}\right)-\mathbf{v} t / \delta^{2}, \quad t \geq 0
$$

converge, as $\delta \downarrow 0$, as continuous stochastic processes, to a zero mean Brownian motion starting at $\mathbf{x}$ with the covariance matrix $\mathbf{D}=\left[D_{i, j}\right]$ given by the Kubo-Taylor formula

$$
D_{i j}=\frac{1}{2} \int_{0}^{+\infty}\left[R_{i j}(\mathbf{v} t)+R_{j i}(\mathbf{v} t)\right] d t, \quad i, j=, \ldots, d .
$$

Here $\mathbf{R}(\mathbf{x})=\left[R_{i j}(\mathbf{x})\right]$ is the covariance matrix of the field $\mathbf{F}(\mathbf{x})$, that is, $R_{i j}(\mathbf{x})=\mathbb{E}\left[F_{i}(\mathbf{x}) F_{j}(\mathbf{0})\right]$.

In the present paper we are interested in the situation when the correlation tensor decays slowly in space so that the diffusion matrix given by the Kubo-Taylor formula is infinite and the standard diffusion limit may fail. More precisely, we consider Gaussian fields which are isotropic and satisfy locally the self-similarity hypothesis. This means that the two-point correlation tensor satisfies

$$
R_{i j}(\mathbf{x})=\int_{\mathbb{R}^{d}} \mathrm{e}^{i \mathbf{k} \cdot \mathbf{x}} \hat{R}_{i j}(\mathbf{k}) d \mathbf{k}
$$

with the power spectrum

$$
\hat{R}_{i j}(\mathbf{k})=\frac{a(|\mathbf{k}|)}{|\mathbf{k}|^{2 \alpha+d-2}} \Gamma_{i j}(\hat{\mathbf{k}}), \quad i, j=, \ldots, d .
$$

Here $a(|\mathbf{k}|) \geq 0$ is a non-negative bounded, measurable function, supported in a finite ball $\{|\mathbf{k}| \leq K\}$ for some $K>0$, continuous at $\mathbf{k}=0$ and with $a(0)=1$. The factor $\Gamma_{i j}(\hat{\mathbf{k}}):=\delta_{i j}-\hat{k}_{i} \hat{k}_{j}$, where $\hat{\mathbf{k}}=\left(\hat{k}_{1}, \ldots, \hat{k}_{d}\right):=\mathbf{k} /|\mathbf{k}|$, ensures incompressibility of the flow. To guarantee the integrability of the spectrum we assume that $\alpha<1$. The rate of decay of the correlations of the field is then given by

$$
R_{i j}(\mathbf{x}) \sim|\mathbf{x}|^{2 \alpha-2}, \quad \text { for }|\mathbf{x}| \gg 1 .
$$

A simple calculation shows that in this situation the diffusion matrix given by the Kubo formula (1.3) is infinite, provided that $1 / 2<\alpha<1$. The goal of this paper is to find the proper time scaling for the tracer trajectory when the Kubo-Taylor formula diverges. In other words we are looking for $H$ such that for any $\rho>0$

$$
\lim _{\delta \rightarrow 0+} \mathbb{E}\left|\mathbf{y}\left(t / \delta^{2 H(1+\rho)} ; \mathbf{x}\right)\right|^{2}=+\infty \quad \text { and } \quad \lim _{\delta \rightarrow 0+} \mathbb{E}\left|\mathbf{y}\left(t / \delta^{2 H(1-\rho)} ; \mathbf{x}\right)\right|^{2}=0 .
$$

The result of Kesten and Papanicolaou, see Theorem 4 of [17, guarantees that for $\mathbf{R}(\mathbf{x})$, which is decaying sufficiently fast, or, equivalently, for $\alpha<-N$ with a large enough $N>0$ one has $H=1$. Note, however, that the Kubo-Taylor formula (1.3) itself makes sense for any $\alpha<1 / 2$, that is, in a much larger range of $\alpha$. We shall prove rigorously in Section 3 below that then indeed $H=1$ for $\alpha<1 / 2$, with a slightly modified definition of the limit appearing in condition (1.7).

According to (1.6), increasing the value of $\alpha$ leads to strengthening of the correlations of the corresponding field (and degraded mixing properties). We shall prove, see Theorems 3.1 and 3.2 below, that the level $\alpha=1 / 2$ is critical in the sense that the value of the exponent $H$ changes at $\alpha=1 / 2$ from $H=1 / 2$ for $\alpha<1 / 2$ to $H=1 /(2 \alpha)<1$ for $1 / 2<\alpha<1$.

We also present a formal argument which provides more refined information on the process $\mathbf{y}(t)$ for $\alpha \in(1 / 2,1)$. In addition to identifying the time scale $t \sim O\left(\delta^{-2 H}\right), H=1 /(2 \alpha)$ on which $\mathbf{y}(t)$ behaves non-trivially, we are able to compute formally the limit of the process

$$
\mathbf{y}_{\delta}(t)=\mathbf{y}\left(t / \delta^{2 H} ; \mathbf{x}\right)=\mathbf{x}\left(t / \delta^{2 H} ; \mathbf{x}\right)-\mathbf{v} t / \delta^{2 H}
$$


as $\delta \rightarrow 0$. It turns out that the limit of $\mathbf{y}_{\delta}(t)$ is a super-diffusive fractional Brownian motion $B_{\alpha}(t)$. This should be contrasted with a standard Brownian motion limit for the process $\mathbf{y}\left(t / \delta^{2}\right)$ obtained in 17] for rapidly decorrelating fields $\mathbf{F}(\mathbf{x})$. We recall that a Gaussian, continuous trajectory, $\mathbb{R}^{d}$-valued process $\left(B_{\alpha}(t)\right)_{t \geq 0}$ is called a fractional Brownian motion $(\mathrm{FBM})$ with Hurst exponent $\alpha \in(0,1)$ and the covariance matrix $\mathbf{D}$ if for any $t, h \geq 0$ the (normal) random vector $B_{\alpha}(t+h)-B_{\alpha}(t)$ has mean zero and the covariance $\mathbf{D} h^{2 \alpha}$. In particular, $\mathbb{E}\left(B_{\alpha}(t) \otimes B_{\alpha}(t)\right)=\mathbf{D} t^{2 \alpha}$ and the tracer propagation is close to ballistic as $\alpha \uparrow 1$ and correlations persist at infinity. Note that for $\alpha=1 / 2$ the FBM becomes the "usual" Brownian motion matching the result of [17] for rapidly decayng correlations.

Anomalous diffusive behavior of passive scalars has been studied extensively in the physics literature - we mention [9, 10, 14, 20, 25, 26] without any attempt at completeness. However, the majority of physical papers consider time-dependent (typically white in time) random flows with a non-zero diffusivity. Super-diffusive limits for particles in a random flow have been also obtained rigorously in various situations - for instance, in [2, 3, 4, 5] for the random shear layer flows, in [1]. for time-dependent random flows, in [18, for a mean zero, random flow with a positive molecular diffusivity and in 7, 22, for multiscale periodic flows. The novel aspect of the present paper is that the flow is time-independent and the molecular diffusivity is equal to zero.

The paper is organized as follows. The aforementioned formal derivation of the fractional Brownian motion limit for $\alpha \in(1 / 2,1)$ is presented in Section 2. We describe in Section 3 upper and lower bounds for the mean square displacement of trajectories that show the super-diffusive scaling for $\alpha \in(1 / 2,1)$ and diffusive behavior for $\alpha<1 / 2$. The main results are Theorems 3.1 and 3.2 they are proved in Sections 4 and 5. Some auxiliary bounds are proved in Section 6. Appendix $\mathrm{A}$ contains a brief review of multiple stochastic integration.

Acknowledgment. LR was supported in part by an Alfred P. Sloan fellowship. This work has been also supported by ONR and NSF grant DMS-0604687.

\section{A formal limit for the advection equation}

\section{The advection equation}

We present here a formal computation that leads to a FBM limit for the solution of the advection equation

$$
\frac{\partial \phi_{\delta}}{\partial t}+(\mathbf{v}+\delta \mathbf{F}(\mathbf{x})) \cdot \nabla_{\mathbf{x}} \phi_{\delta}=0, \quad \phi_{\delta}(0, \mathbf{x})=u_{0}(\mathbf{x}) .
$$

Here $\mathbf{v} \in \mathbb{R}^{d}$ is a fixed mean flow with $|\mathbf{v}|=1$ and $\mathbf{F}(\mathbf{x})$ is a spatially homogeneous Gaussian random field with the two-point correlation tensor $\left[R_{i j}(\mathbf{x})\right]$ of the form (1.4) and the power spectrum as in (1.5)). We can write then $\mathbf{F}(\mathbf{x})=\int e^{i \mathbf{x} \cdot \mathbf{k}} \hat{\mathbf{F}}(d \mathbf{k})$, where the spectral measure $\hat{F}(d \mathbf{k})$ satisfies

$$
\mathbb{E}\left[\hat{F}_{n}(d \mathbf{k}) \hat{F}_{m}(d \mathbf{p})\right]=\hat{R}_{n m}(\mathbf{k}) \delta(\mathbf{k}+\mathbf{p}) d \mathbf{k} d \mathbf{p} .
$$

We will assume in this section that the exponent $\alpha \in(1 / 2,1)$ as this is the range where we expect the FBM behavior for a rescaled process in the limit $\delta \rightarrow 0$. Let us set $H=1 /(2 \alpha)$ and introduce the rescaled time $t^{\prime}=\delta^{2 H} t$ and the moving frame $\mathbf{x}^{\prime}=\mathbf{x}+\mathbf{v} t$ as well as the new unknown function $u_{\delta}\left(t^{\prime}, \mathbf{x}^{\prime}\right)=\phi_{\delta}\left(\frac{t^{\prime}}{\delta^{2 H}}, \mathbf{x}^{\prime}-\mathbf{v} \frac{t^{\prime}}{\delta^{2 H}}\right)$. Then (2.1) becomes in the new variables

$$
\frac{\partial u_{\delta}}{\partial t^{\prime}}+\delta^{1-2 H} \mathbf{F}\left(\mathbf{x}^{\prime}-\mathbf{v} \frac{t^{\prime}}{\delta^{2 H}}\right) \cdot \nabla_{\mathbf{x}^{\prime}} u_{\delta}=0, \quad u_{\delta}\left(0, \mathbf{x}^{\prime}\right)=u_{0}\left(\mathbf{x}^{\prime}\right) .
$$

For convenience we introduce $\mathbf{G}(t, \mathbf{x})=-\mathbf{F}(\mathbf{x}-\mathbf{v} t)$ and drop the primes, arriving at

$$
\frac{\partial u_{\delta}}{\partial t}-\delta^{1-2 H} \mathbf{G}\left(\frac{t}{\delta^{2 H}}, \mathbf{x}\right) \cdot \nabla_{\mathbf{x}} u_{\delta}=0, u_{\delta}(0, \mathbf{x})=u_{0}(\mathbf{x}) .
$$


The main goal of this section is to argue formally that $\mathbb{E}\left[u_{\delta}(t, \mathbf{x})\right]$ converges, as $\delta \rightarrow 0$, to

$$
\bar{u}(t, \mathbf{x})=\mathbb{E}\left[u_{0}\left(\mathbf{x}+B_{D}(t)\right)\right] .
$$

Here $B_{D}(t)$ is the FBM with exponent $\alpha$ and covariance matrix $\mathbf{D}=\left[D_{i j}\right]$, where

$$
D_{i j}=\frac{1}{2 \alpha^{2}} \int \frac{e^{i \mathbf{k} \cdot \mathbf{v}}}{|\mathbf{k}|^{2 \alpha+d-2}} \Gamma_{i j}(\hat{\mathbf{k}}) d \mathbf{k} .
$$

We will establish this limit by evaluating term-wise the expectation of the formal infinite Duhamel expansion for the solution of (2.4) and computing the limit of the main term. We will neither attempt to justify the expansion, nor try to estimate the error produced by the terms in the expansion which are formally of a smaller order. The main difference with the corresponding calculation in the case when correlations are rapidly decaying (the Brownian motion limit case) is that all Feynman diagrams contribute to the limit and not only the so-called ladder diagrams.

\section{An infinite expansion for the solution}

We rewrite (2.4) as an integral equation:

$$
u_{\delta}(t, \mathbf{x})=u_{0}(\mathbf{x})+\delta^{1-2 H} \int_{0}^{t} \mathbf{G}\left(\frac{s_{1}}{\delta^{2 H}}, \mathbf{x}\right) \cdot \nabla_{\mathbf{x}} u_{\delta}\left(s_{1}, \mathbf{x}\right) d s_{1} .
$$

Iterating (2.7) by substituting for $u_{\delta}\left(s_{1}, \cdot\right)$ appearing on the right side we obtain a formal expansion

$$
u_{\delta}(t, \mathbf{x})=\sum_{n=0}^{+\infty} \delta^{(1-2 H) n} \int_{\Delta_{n}(t)} \mathbf{G}^{(n)}\left(\frac{s_{1}}{\delta^{2 H}}, \ldots, \frac{s_{n}}{\delta^{2 H}}, \mathbf{x}\right) d s_{1} \ldots d s_{n}
$$

Here, we have set $\mathbf{G}^{(0)}(\mathbf{x}):=u_{0}(\mathbf{x})$ and, assuming that $\mathbf{G}^{(n)}\left(s_{1}, \ldots, s_{n}, \mathbf{x}\right)$ has been already defined for some $n \geq 1$, we let

$$
\mathbf{G}^{(n+1)}\left(s_{1}, \ldots, s_{n}, s_{n+1}, \mathbf{x}\right):=\mathbf{G}\left(s_{n+1}, \mathbf{x}\right) \cdot \nabla_{\mathbf{x}} \mathbf{G}^{(n)}\left(s_{1}, \ldots, s_{n}, \mathbf{x}\right) .
$$

The time integration region appearing in (2.8) is the simplex

$$
\Delta_{n}(t):=\left[\left(s_{1}, \ldots, s_{n}\right) \in \mathbb{R}^{n}: 0 \leq s_{n} \leq \ldots \leq s_{1} \leq t\right] .
$$

Passing to the Fourier transform in the definition of $G^{(n)}$ and using induction we arrive at an explicit expression

$$
\mathbf{G}^{(n)}\left(s_{1}, \ldots, s_{n}, \mathbf{x}\right)=i^{n} \int e^{-i \mathbf{v} \cdot\left(\mathbf{k}_{1} s_{1}+\ldots+\mathbf{k}_{n} s_{n}\right)} e^{i \mathbf{x} \cdot\left(\mathbf{k}_{0}+\ldots+\mathbf{k}_{n}\right)} \prod_{p=1}^{n}\left[\left(\sum_{l=0}^{p-1} \mathbf{k}_{l}\right) \cdot \hat{\mathbf{F}}\left(d \mathbf{k}_{p}\right)\right] \hat{u}_{0}\left(\mathbf{k}_{0}\right) d \mathbf{k}_{0}
$$

Next, we take formally the expectation of the infinite series in (2.8) term-wise and obtain

$$
\mathbb{E}\left[u_{\delta}(t, \mathbf{x})\right]=\sum_{n=0}^{+\infty} I_{n}(\delta)(t, \mathbf{x})
$$

where

$$
I_{n}(\delta)(t, \mathbf{x})=\delta^{2(1-2 H) n} \int_{\Delta_{2 n}(t)} \mathbb{E} \mathbf{G}^{(2 n)}\left(\frac{s_{1}}{\delta^{2 H}}, \ldots, \frac{s_{2 n}}{\delta^{2 H}}, \mathbf{x}\right) \prod_{i=1}^{2 n} d s_{i}
$$

We have used above the fact that the expectation of a product of an odd number of centered Gaussian variables equals zero. The next step is to evaluate each of the terms $I_{n}(\delta)$. 


\section{The main term in the expansion}

We will compute the individual terms $I_{n}(\delta)$ using Feynman diagrams. Recall that given Gaussian random variables $Y_{1}, \ldots, Y_{2 n}$ the expectation $\mathbb{E}\left(Y_{1} Y_{2} \ldots Y_{2 n}\right)$ is

$$
\mathbb{E}\left(Y_{1} Y_{2} \ldots Y_{2 n}\right)=\sum_{\mathcal{F} \in \mathfrak{F}(n)} \prod_{\widehat{p} q \in \mathcal{F}} \mathbb{E}\left(Y_{p} Y_{q}\right)
$$

Here $\mathfrak{F}(n)$ is the set of all permutations (complete Feynman diagrams) of the elements of $\{1, \ldots, 2 n\}$ such that $\mathcal{F} \circ \mathcal{F}=I d$, and $\mathcal{F}(k) \neq k$ for all $1 \leq k \leq 2 n$. The notation $\widehat{p q} \in \mathcal{F}$ means that $\mathcal{F}(p)=q$ (or that $p$ and $q$ are connected by an edge in the Feynman diagram $\mathcal{F}$ ) and $p<q$.

Using (2.11) in the expression for $I_{n}(\delta)$ we obtain from (2.9) and (2.10) that

$$
\begin{aligned}
& I_{n}(\delta)(t, \mathbf{x})=(-1)^{n} \delta^{2(1-2 H) n} \sum_{\mathcal{F} \in \mathfrak{F}(n)} \sum_{i_{1}, \ldots, i_{2 n}=1}^{d} \int \ldots \int_{\Delta_{2 n}(t)} e^{-i \mathbf{v} \cdot\left(\mathbf{k}_{1} s_{1} \delta^{-2 H}+\ldots+\mathbf{k}_{2 n} s_{2 n} \delta^{-2 H}\right)} \\
& \times e^{i \mathbf{x} \cdot\left(\mathbf{k}_{0}+\mathbf{k}_{1}+\ldots+\mathbf{k}_{2 n}\right)} \prod_{\hat{p} q \in \mathcal{F}}\left\{\left(\sum_{l=0}^{p-1} k_{l, i_{p}}\right)\left(\sum_{j=0}^{q-1} k_{j, i_{q}}\right) \mathbb{E}\left[\hat{F}_{i_{p}}\left(d \mathbf{k}_{p}\right) \hat{F}_{i_{q}}\left(d \mathbf{k}_{q}\right)\right]\right\} \hat{u}_{0}\left(\mathbf{k}_{0}\right) d \mathbf{k}_{0} \prod_{i=1}^{2 n} d s_{i} .
\end{aligned}
$$

After evaluating the expectation above, using (2.2), this expression becomes

$$
\begin{aligned}
& I_{n}(\delta)(t, \mathbf{x})=(-1)^{n} \delta^{2(1-2 H) n} \sum_{i_{1}, \ldots, i_{2 n}=1}^{d} \sum_{\mathcal{F} \in \mathfrak{F}(n)} \int \ldots \int_{\Delta_{2 n}(t)} \prod_{\widehat{p} q \in \mathcal{F}}\left\{\exp \left\{i \mathbf{v} \cdot \mathbf{k}_{p} \frac{s_{q}-s_{p}}{\delta^{2 H}}\right\}\right. \\
& \left.\times\left(k_{0, i_{p}}+\sum_{l=1}^{p-1} k_{l, i_{p}}\right)\left(k_{0, i_{q}}+\sum_{j=1}^{q-1} k_{j, i_{q}}\right) \hat{R}_{i_{p} i_{q}}\left(\mathbf{k}_{p}\right) \delta\left(\mathbf{k}_{p}+\mathbf{k}_{q}\right) d \mathbf{k}_{p} d \mathbf{k}_{q}\right\} e^{i \mathbf{k}_{0} \cdot \mathbf{x}_{\hat{u}_{0}}\left(\mathbf{k}_{0}\right) d \mathbf{k}_{0} \prod_{i=1}^{2 n} d s_{i} .}
\end{aligned}
$$

The leading order term in (2.12) is

$$
\begin{aligned}
& I_{n}^{0}(\delta)(t, \mathbf{x})=(-1)^{n} \delta^{2(1-2 H) n} \sum_{i_{1}, \ldots, i_{2 n}=1}^{d} \sum_{\mathcal{F} \in \mathfrak{F}(n)} \int \ldots \int_{\Delta_{2 n}(t)} \prod_{\hat{p} q \in \mathcal{F}}\left[\exp \left\{i \mathbf{v} \cdot \mathbf{k}_{p} \frac{s_{q}-s_{p}}{\delta^{2 H}}\right\}\right. \\
& \left.\times k_{0, i_{p}} k_{0, i_{q}} \hat{R}_{i_{p} i_{q}}\left(\mathbf{k}_{p}\right) d \mathbf{k}_{p}\right] e^{i \mathbf{k}_{0} \cdot \mathbf{x}} \hat{u}\left(\mathbf{k}_{0}\right) d \mathbf{k}_{0} \prod_{i=1}^{2 n} d s_{i} \\
& =(-1)^{n} \delta^{2(1-2 H) n} \sum_{\mathcal{F} \in \mathfrak{F}(n)} \int_{\Delta_{2 n}(t)} \ldots \prod_{\hat{p} q \in \mathcal{F}}\left[R\left(\frac{s_{q}-s_{p}}{\delta^{2 H}} \mathbf{v}\right) \mathbf{k}_{0} \cdot \mathbf{k}_{0}\right] e^{i \mathbf{k}_{0} \cdot \mathbf{x}} \hat{u}_{0}\left(\mathbf{k}_{0}\right) d \mathbf{k}_{0} \prod_{i=1}^{2 n} d s_{i} .
\end{aligned}
$$

The reason why $I_{n}^{0}(\delta)(t, \mathbf{x})$ is indeed the main contribution to $I_{n}(\delta)(t, \mathbf{x})$ is as follows. Note that the two-point correlation function $R_{i j}(\mathbf{x})$ decays algebraically for large $|\mathbf{x}|$ :

$$
R_{i j}(\mathbf{v} s)=D_{i j}(s) s^{2 \alpha-2},
$$

with the matrix $D_{i j}(s)$, which converges, as $s \rightarrow+\infty$, to

$$
\bar{D}_{i j}=\int \frac{1}{|\mathbf{k}|^{2 \alpha+d-2}} \Gamma_{i j}(\hat{\mathbf{k}}) e^{i \mathbf{k} \cdot \mathbf{v}} d \mathbf{k}
$$


(a more explicit expression for $\bar{D}_{i j}$ using Legendre polynomials may be obtained using the HeckeFunk theorem (see e.g. [15], p. 181) but we will not need it here). Using the above information in (2.13) we obtain:

$$
I_{n}^{0}(\delta)(t, \mathbf{x})=(-1)^{n} \delta^{2(1-2 H) n} \sum_{\mathcal{F} \in \mathfrak{F}(n)} \int_{\Delta_{2 n}(t)} \ldots \int e^{i \mathbf{k}_{0} \cdot \mathbf{x}}\left(\overline{\mathbf{D}} \mathbf{k}_{0} \cdot \mathbf{k}_{0}\right)^{n} \hat{u}\left(\mathbf{k}_{0}\right) d \mathbf{k}_{\widehat{p} q \in \mathcal{F}}\left|\frac{s_{q}-s_{p}}{\delta^{2 H}}\right|^{2 \alpha-2} \prod_{i=1}^{2 n} d s_{i}+o(1) .
$$

where $\overline{\mathbf{D}}=\left[\bar{D}_{i j}\right]$. However, and this is the crucial point in this calculation, as $H=1 /(2 \alpha)$ we see that

$$
2(1-2 H) n-2 H n(2 \alpha-2)=0
$$

and the powers of $\delta$ exactly cancel each other. We conclude that $I_{n}^{0}(\delta)(t, \mathbf{x})=J_{n}(t, \mathbf{x})+o(1)$, with

$$
J_{n}(t, \mathbf{x})=(-1)^{n} \sum_{\mathcal{F} \in \mathfrak{F}(n)} \int_{\Delta_{2 n}(t)} \ldots \int_{\int^{i}} e^{i \mathbf{k}_{0} \cdot \mathbf{x}}\left(\overline{\mathbf{D}} \mathbf{k}_{0} \cdot \mathbf{k}_{0}\right)^{n} \prod_{\hat{p} q \in \mathcal{F}}\left|s_{q}-s_{p}\right|^{2 \alpha-2} \hat{u}_{0}\left(\mathbf{k}_{0}\right) d \mathbf{k}_{0} \prod_{i=1}^{2 n} d s_{i} .
$$

In particular, $I_{n}^{0}(\delta)(t, \mathbf{x})$ is of order $O(1)$, as $\delta \rightarrow 0$. On the other hand, all the other terms in (2.12) lead to expressions similar to (2.13) but with $R_{i j}(\mathbf{x})$ replaced by its (possibly higher order) derivatives with respect to some of the spatial variables. However, derivatives of $R_{i j}(\mathbf{x})$ decay faster than $|\mathbf{x}|^{2-2 \alpha}$ for large $|\mathbf{x}|$ - hence these terms produce a too high power of $\delta$ as a factor, and vanish (at least, term-wise) in the limit $\delta \rightarrow 0$.

\section{Interpretation in terms of the fractional Brownian motion}

It remains now to relate $J_{n}(t, \mathbf{x})$ to the fractional Brownian motion and sum all these terms. Note that the function

$$
f\left(s_{1}, \ldots, s_{2 n}\right):=\sum_{\mathcal{F} \in \mathfrak{F}(n)} \prod_{\widehat{p q} \in \mathcal{F}}\left|s_{p}-s_{q}\right|^{2 \alpha-2}
$$

is symmetric in all of its arguments, that is, $f\left(s_{1}, \ldots, s_{2 n}\right)=f\left(s_{\pi(1)}, \ldots, s_{\pi(2 n)}\right)$, where $\pi$ is an arbitrary permutation of $\{1,2 \ldots, n\}$. Using this fact we can rewrite $J_{n}(t, \mathbf{x})$ in the form

$$
J_{n}(t, \mathbf{x})=\frac{(-1)^{n}}{(2 n) !} \sum_{\mathcal{F} \in \mathfrak{F}(n)} \int_{0}^{t} \ldots \int_{0}^{t} \prod_{\widehat{p} q \in \mathcal{F}}\left|s_{p}-s_{q}\right|^{2 \alpha-2} \prod_{i=1}^{2 n} d s_{i} \int e^{i \mathbf{k}_{0} \cdot \mathbf{x}}\left(\overline{\mathbf{D}} \mathbf{k}_{0}, \mathbf{k}_{0}\right)^{n} \hat{u}_{0}\left(\mathbf{k}_{0}\right) d \mathbf{k}_{0} .
$$

A simple but useful observation is that

$$
\sum_{\mathcal{F} \in \mathfrak{F}(n)} \prod_{p}\left|s_{p}-s_{q}\right|^{2 \alpha-2}=c_{\alpha}^{2 n} \mathbb{E}\left[\prod_{p=1}^{2 n} \int_{-\infty}^{\infty} \frac{\mathrm{e}^{i k_{p} s_{p}}}{\left|k_{p}\right|^{\alpha-1 / 2}} w\left(d k_{p}\right)\right],
$$

where $w\left(d k_{1}\right), \ldots, w\left(d k_{2 n}\right)$ are independent Gaussian white noises and $c_{\alpha}>0$ is given by

$$
c_{\alpha}:=\left(\frac{\Gamma(2 \alpha-1) \sin (\pi \alpha)}{\pi}\right)^{1 / 2}
$$

This follows from the fact that

$$
\mathbb{E}\left[\int_{-\infty}^{\infty} \frac{\mathrm{e}^{i k_{1} s}}{\left|k_{1}\right|^{\alpha-1 / 2}} w\left(d k_{1}\right) \int_{-\infty}^{\infty} \frac{\mathrm{e}^{i k_{2} r}}{\left|k_{2}\right|^{\alpha-1 / 2}} w\left(d k_{2}\right)\right]=\int_{-\infty}^{\infty} \frac{\mathrm{e}^{i k_{1}(s-r)}}{\left|k_{1}\right|^{2 \alpha-1}} d k_{1}=c_{\alpha}^{-2}|s-r|^{2 \alpha-2}
$$


for

$$
\int_{-\infty}^{\infty} \frac{e^{i k}}{|k|^{2 \alpha-1}} d k=2 \int_{0}^{+\infty} \frac{\cos k}{k^{2 \alpha-1}} d k=\frac{\pi}{\Gamma(2 \alpha-1) \sin (\pi \alpha)}
$$

The last equality follows e.g. from 3), [539] of [13]. Hence, $J_{n}(t, \mathbf{x})$ has a representation

$$
J_{n}(t, \mathbf{x})=\frac{(-1)^{n}}{(2 n) !} \int_{0}^{t} \cdots \int_{0}^{t} \mathbb{E}\left[\prod_{p=1}^{2 n} \int \frac{\mathrm{e}^{i k_{p} s_{p}}}{\left|k_{p}\right|^{\alpha-1 / 2}} w\left(d k_{p}\right)\right] \prod_{i=1}^{2 n} d s_{i} \int e^{i \mathbf{k}_{0} \cdot \mathbf{x}}\left|c_{\alpha}\right|^{2 n}\left(\overline{\mathbf{D}} \mathbf{k}_{0}, \mathbf{k}_{0}\right)^{n} \hat{u}_{0}\left(\mathbf{k}_{0}\right) d \mathbf{k}_{0} .
$$

Performing now the integrations with respect to $s_{i}$ on the right side we conclude that

$$
J_{n}(t, \mathbf{x})=\frac{(-1)^{n}}{(2 n) !} \mathbb{E}\left[\prod_{p=1}^{2 n} \int \frac{\mathrm{e}^{i k_{p} t}-1}{i k_{p}\left|k_{p}\right|^{\alpha-1 / 2}} w\left(d k_{p}\right)\right] \int\left|c_{\alpha}\right|^{2 n}\left(\overline{\mathbf{D}} \mathbf{k}_{0}, \mathbf{k}_{0}\right)^{n} \hat{u}_{0}\left(\mathbf{k}_{0}\right) e^{i \mathbf{k}_{0} \cdot \mathbf{x}} d \mathbf{k}_{0} .
$$

Next, using the harmonizable representation of the standard fractional Brownian motion, see Proposition 7.2 .8 , p. 328 of [24], we obtain

$$
J_{n}(t, \mathbf{x})=\frac{(-1)^{n}}{(2 n) !} c^{2 n}\left|c_{\alpha}\right|^{2 n} \mathbb{E} B_{\alpha}(t)^{2 n} \int e^{i \mathbf{k}_{0} \cdot \mathbf{x}}\left(\overline{\mathbf{D}} \mathbf{k}_{0}, \mathbf{k}_{0}\right)^{n} \hat{u}_{0}\left(\mathbf{k}_{0}\right) d \mathbf{k}_{0}
$$

Here $B_{\alpha}(t)$ is a fractional Brownian motion with the Hurst exponent $\alpha$ and

$$
c:=\left(\frac{\pi}{\alpha \Gamma(2 \alpha) \sin (\alpha \pi)}\right)^{1 / 2}=\left(\frac{\pi}{2 \alpha^{2} \Gamma(2 \alpha-1) \sin (\alpha \pi)}\right)^{1 / 2}
$$

Setting $b:=c c_{\alpha}=1 /(\alpha \sqrt{2})$ we may now re-write $J_{n}$ as

$$
\begin{aligned}
J_{n}(t, \mathbf{x}) & =\frac{(-1)^{n}(2 n-1) ! !}{(2 n) !} t^{2 n \alpha} b^{2 n} \int e^{i \mathbf{k}_{0} \cdot \mathbf{x}}\left(\overline{\mathbf{D}} \mathbf{k}_{0}, \mathbf{k}_{0}\right)^{n} \hat{u}_{0}\left(\mathbf{k}_{0}\right) d \mathbf{k}_{0} \\
& =\frac{1}{n !} \int e^{i \mathbf{k}_{0} \cdot \mathbf{x}}\left[-\frac{b^{2} t^{2 \alpha}}{2}\left(\overline{\mathbf{D}} \mathbf{k}_{0}, \mathbf{k}_{0}\right)\right]^{n} \hat{u}_{0}\left(\mathbf{k}_{0}\right) d \mathbf{k}_{0} .
\end{aligned}
$$

Coming back to (2.10) we see that, as $\delta \rightarrow 0$,

$$
\begin{aligned}
\mathbb{E} u_{\delta}(t, \mathbf{x}) \rightarrow \bar{u}(t, \mathbf{x})= & \sum_{n=0}^{\infty} J_{n}(t, \mathbf{x})=\int \exp \left\{i \mathbf{k}_{0} \cdot \mathbf{x}-\frac{b^{2} t^{2 \alpha}}{2}\left(\overline{\mathbf{D}} \mathbf{k}_{0}, \mathbf{k}_{0}\right)\right\} \hat{u}_{0}\left(\mathbf{k}_{0}\right) d \mathbf{k}_{0} \\
& =\int \exp \left\{i\left(\mathbf{x}+\mathbf{B}_{\mathbf{D}}(t)\right) \cdot \mathbf{k}_{0}\right\} \hat{u}_{0}\left(\mathbf{k}_{0}\right) d \mathbf{k}_{0}=\mathbb{E}\left[u_{0}\left(\mathbf{x}+\mathbf{B}_{\mathbf{D}}(t)\right)\right],
\end{aligned}
$$

where $\mathbf{B}_{\mathbf{D}}(t)$ is a $d$-dimensional fractional Brownian motion with the exponent $\alpha$ and the covariance matrix $\mathbf{D}=\overline{\mathbf{D}} /\left(2 \alpha^{2}\right)$. Therefore, we have formally established that (2.5) gives the limit of $\mathbb{E} u_{\delta}(t, \mathbf{x})$.

In terms of the characteristics

$$
\frac{d \mathbf{X}(t ; \mathbf{x})}{d t}=\mathbf{v}+\delta \mathbf{F}(\mathbf{X}(t ; \mathbf{x})), \quad \mathbf{X}(0 ; \mathbf{x})=\mathbf{x},
$$

for the original advection problem (2.1) we have formally argued that the one dimensional statistics of the process

$$
\mathbf{y}(t ; \mathbf{x})=\mathbf{X}\left(\frac{t}{\delta^{2 H}} ; \mathbf{x}\right)-\frac{\mathbf{v} t}{\delta^{2 H}},
$$

with $H=1 /(2 \alpha)$, converge, as $\delta \rightarrow 0$, to the corresponding statistics of a fractional Brownian motion with the exponent $\alpha$ and diffusion matrix $\mathbf{D}$ given by (2.6). In the next section we will show rigorously that indeed the process $\mathbf{y}(t)$ behaves trivially (either vanishes or $\mathbb{E}\left[|\mathbf{y}(t)|^{2}\right]$ tends to infinity) on all time scales but $t \sim O\left(\delta^{-2 H}\right)$. 


\section{The main results: rigorous bounds for the trajectories}

\section{Preliminaries}

The probability space. Suppose that $m$ is a positive integer and $\vartheta_{\rho}(\mathbf{x}):=\left(1+|\mathbf{x}|^{2}\right)^{-\rho}, \mathbf{x} \in \mathbb{R}^{d}$, where $\rho>d / 2$. Let $\Omega$ be the Hilbert space of $d$-dimensional incompressible vector fields that is the completion of the space $C_{0, \text { div }}^{\infty}:=\left\{\omega \in C_{0}^{\infty}\left(\mathbb{R}^{d} ; \mathbb{R}^{d}\right): \nabla_{\mathbf{x}} \cdot \omega=0\right\}$ with respect to the norm

$$
\|\omega\|_{\Omega}^{2}:=\int_{\mathbb{R}^{d}}\left(|\omega(\mathbf{x})|^{2}+\left|\nabla_{\mathbf{x}} \omega(\mathbf{x})\right|^{2}+\cdots+\left|\nabla_{\mathbf{x}}^{m} \omega(\mathbf{x})\right|^{2}\right) \vartheta_{\rho}(\mathbf{x}) d \mathbf{x} .
$$

We shall assume that $m>d / 2+1$ so that any $\omega \in \Omega$ is of $C^{1}$ class of regularity by the Sobolev embedding theorem.

The random field. The random field is set to be simply $\mathbf{F}(\mathbf{x} ; \omega):=\omega(\mathbf{x})$. Denote also $\mathbf{F}(\omega)=$ $\left(F_{1}(\omega), \ldots, F_{d}(\omega)\right):=\omega(\mathbf{0})$. We suppose that $\mathbb{P}$ is a Borel measure given on $\Omega$ that satisfies the following hypotheses:

(H1) it is Gaussian, that is, for any $N \geq 1, \mathbf{x}_{1}, \ldots, \mathbf{x}_{N} \in \mathbb{R}^{d}$ we have $\mathbf{F}\left(\mathbf{x}_{1}\right), \ldots, \mathbf{F}\left(\mathbf{x}_{N}\right)$ is a Gaussian, $N d$-dimensional random vector,

(H2) it is centered and homogeneous, that is, $\mathbf{F}$ is of mean zero and the two-point correlation matrix depends only on the relative position of the points:

$$
\int F_{i}(\mathbf{z} ; \omega) F_{j}(\mathbf{y} ; \omega) d \mathbb{P}(\omega)=R_{i j}(\mathbf{z}-\mathbf{y})
$$

where $\mathbf{R}(\mathbf{x})=\left[R_{i j}(\mathbf{x})\right]$ is given by (1.4). As the measure $\mathbb{P}$ is Gaussian, this condition guarantees that $\mathbb{P}$ is invariant with respect to any spatial shift transformation $\tau_{\mathbf{x}}: \Omega \rightarrow \Omega$, $\mathbf{x} \in \mathbb{R}^{d}$ defined by $\tau_{\mathbf{x}} \omega(\mathbf{z}):=\omega(\mathbf{x}+\mathbf{z})$. The existence of such a measure on $\Omega$ is guaranteed e.g. by the results of [12, see Section 2.3. Thanks to the assumed form of the power spectrum we may suppose that the realizations of the velocity field are $\mathbb{P}$-a.s. analytic in the $\mathbf{x}$ variable, see e.g. 6 . We will denote by $\mathbb{E}$ the mathematical expectation with respect to the measure $\mathbb{P}$.

\section{The main results}

We consider the particle trajectory given as the solution to (2.17) with the starting point $\mathbf{x}=\mathbf{0}$ and with the random field $\mathbf{F}(\mathbf{x})$ constructed in the previous section, and define the particle deviation from the mean position $\mathbf{y}(t)=\mathbf{X}(t ; \mathbf{0})-\mathbf{v} t$. We also introduce

$$
Y_{i}(t):=\int_{0}^{t} \mathbb{E}\left[y_{i}(s)\right]^{2} d s, \quad i=1, \ldots, d .
$$

Suppose that the times $T_{\delta}>0$ are such that $\lim _{\delta \rightarrow 0+} T_{\delta}=+\infty$. Define the Cesaro limit of the mean square of the fluctuation amplitude as

$$
C-\lim _{\delta \rightarrow 0+} \mathbb{E}\left|\mathbf{y}\left(T_{\delta}\right)\right|^{2}:=\lim _{\delta \rightarrow 0+} \frac{1}{T_{\delta}} Y\left(T_{\delta}\right),
$$

provided that the limit on the right hand side exists, whether it is finite, or not.

We will distinguish two cases: as we have mentioned in the Introduction, when $\alpha \in(1 / 2,1)$ the Kubo-Taylor formula (1.3) diverges and we expect a behavior different from the usual diffusive limit. On the other hand, when $\alpha<1 / 2$ the diffusion coefficient given by (1.3) remains finite and the usual diffusive behavior would not be surprising. 
The case $\alpha \in(1 / 2,1)$

We have argued formally in Section 2 that in this range the process $\mathbf{y}(t)$ converges on the time scale $t \sim O\left(\delta^{-2 H}\right), H=1 /(2 \alpha)$ to a fractional Brownian motion with the exponent $\alpha$. Our first result confirms the predicted time-scale in this range of the parameter $\alpha$.

Theorem 3.1 Suppose that $t>0, \alpha \in(1 / 2,1)$ and $\rho>0$. Let $T_{\delta}^{+}:=\delta^{-2 H(1+\rho)} t^{1+\rho}$ and $T_{\delta}^{-}:=$ $\delta^{-2 H(1-\rho)} t^{1-\rho}$. Under the above assumptions about the random field $\mathbf{F}(\mathbf{x})$ we have

$$
C-\lim _{\delta \rightarrow 0+} \mathbb{E}\left|\mathbf{y}\left(T_{\delta}^{+}\right)\right|^{2}=+\infty
$$

and

$$
\lim _{\delta \rightarrow 0+} \mathbb{E}\left|\mathbf{y}\left(T_{\delta}^{-}\right)\right|^{2}=0
$$

The case $\alpha<1 / 2$

In this case we expect that $\mathbf{y}(t)$ behaves diffusively on the time scale $t \sim O\left(\delta^{-2}\right)$, as in the situation when the correlation tensor decays rapidly in space, that is, when $\alpha$ is very negative. This time scale is confirmed by the next theorem.

Theorem 3.2 Suppose that $\alpha<1 / 2$. Then, for arbitrary $t, \rho>0$ we have

$$
C-\lim _{\delta \rightarrow 0+} \delta^{-\rho} \mathbb{E}\left|\mathbf{y}\left(t \delta^{-2}\right)\right|^{2}=+\infty
$$

and

$$
\lim _{\delta \rightarrow 0+} \mathbb{E}\left|\mathbf{y}\left(t \delta^{-2(1-\rho)}\right)\right|^{2}=0 .
$$

Theorems 3.1 and 3.2 are proved in Sections 4 and 5 respectively.

\section{The proof of Theorem 3.1}

\section{The lower bound}

We prove the lower bound (3.3). This is done with the help of a general lower bound, which relates the long time behavior of the trajectory to the behavior of the resolvent near the border of the spectrum $\lambda=0$. In order to formulate it let us begin with some preliminary definitions.

The basic spaces. For any $p \in[1,+\infty)$ and $\phi \in L^{p}(\mathbb{P})$ we adopt the notation $D_{k} \phi:=$ $\left.\frac{d}{d h} \phi\left(\tau_{h} \mathbf{e}_{k} \omega\right)\right|_{h=0}$, where $\mathbf{e}_{k}, k=1, \cdots, d$ is the $k$-th vector of the canonical basis in $\mathbb{R}^{d}$. The derivatives are understood in the $L^{p}$ sense. Let $W^{p, m}$ be the Banach space consisting of those elements $\phi \in$ $L^{p}(\mathbb{P})$, for which

$$
\|\phi\|_{p, m}^{p}:=\sum_{i_{1}+\cdots+i_{d} \leq m}\left\|D_{1}^{i_{1}} \cdots D_{d}^{i_{d}} \phi\right\|_{L^{p}}^{p}<+\infty
$$

We set

$$
\mathcal{C}:=\bigcap_{1<p<+\infty} W^{p, 2}
$$

The spectral measure. The spectral theorem (see e.g. Theorem 1.4.2, p. 18 of [23]) implies that there exists a complex vector valued spectral measure $\hat{\mathbf{F}}(\cdot)=\left(\hat{F}_{1}(\cdot), \ldots, \hat{F}_{d}(\cdot)\right)$ defined over $\left(\mathbb{R}^{d}, \mathcal{B}\left(\mathbb{R}^{d}\right)\right)$ whose components take values in $L^{2}(\mathbb{P})$ such that

$$
\mathbf{F}(\mathbf{x})=\int \mathrm{e}^{i \mathbf{x} \cdot \mathbf{k}} \hat{\mathbf{F}}(d \mathbf{k})
$$


The spectral measure is Gaussian, that is, for any set $A \in \mathcal{B}\left(\mathbb{R}^{d}\right)$ the $2 d$-dimensional random vector $(\operatorname{Re} \hat{\mathbf{F}}(A), \operatorname{Im} \hat{\mathbf{F}}(A))$ is Gaussian. Since the random field (4.2) is real vector valued we must have $\hat{\mathbf{F}}^{*}(d \mathbf{k})=\hat{\mathbf{F}}(-d \mathbf{k})$. The corresponding structure measure equals

$$
\mathbb{E}\left[\hat{F}_{i}(d \mathbf{k}) \hat{F}_{j}^{*}\left(d \mathbf{k}^{\prime}\right)\right]=\hat{R}_{i j}(\mathbf{k}) \delta\left(\mathbf{k}-\mathbf{k}^{\prime}\right) d \mathbf{k} d \mathbf{k}^{\prime}, \quad i, j=1, \ldots, d .
$$

The Hermite polynomials. Suppose that $\psi=\left(\psi_{1}, \ldots, \psi_{d}\right)$, where $\psi_{i} \in C_{0}^{\infty}\left(\mathbb{R}^{d}\right), i=1, \ldots, d$ are complex valued, even functions, that is, $\psi_{i}(-\mathbf{k})=\psi_{i}^{*}(\mathbf{k})$. We write

$$
\int \psi(\mathbf{k}) \cdot \hat{\mathbf{F}}(d \mathbf{k}):=\sum_{i=1}^{d} \int \psi_{i}(\mathbf{k}) \hat{F}_{i}(d \mathbf{k}) .
$$

Denote by $H$ the subspace of $L^{2}(\mathbb{P})$ obtained by taking the closure of the linear span of the elements of the form (4.4). It is a Gaussian Hilbert space in the sense of Definition 1.2 p. 4 of 16. We can define then, see Definition 2.1 of ibid., the space of the $n$-th degree polynomials $\mathcal{P}_{n}$ as the $L^{2}$-closure of all the elements of the form $p\left(\phi_{1}, \ldots, \phi_{k}\right)$, where $p(\cdot)$ is polynomial of at most $n$-th degree with real valued coefficients and $\phi_{i} \in H, i=1, \ldots, k$. It can easily be seen that $\mathcal{P}_{n}$ can be also characterized as the $L^{2}$-closure of the space spanned by

$$
\int \ldots \int \psi\left(\mathbf{k}_{1}, \ldots, \mathbf{k}_{n}\right) \cdot \hat{\mathbf{F}}\left(d \mathbf{k}_{1}\right) \otimes \ldots \otimes \hat{\mathbf{F}}\left(d \mathbf{k}_{n}\right)
$$

where $\psi:\left(\mathbb{R}^{d}\right)^{n} \rightarrow\left(\mathbb{C}^{d}\right)^{n}$ is even, that is, $\psi\left(-\mathbf{k}_{1}, \ldots,-\mathbf{k}_{n}\right)=\psi^{*}\left(\mathbf{k}_{1}, \ldots, \mathbf{k}_{n}\right)$, - see the Appendix for the definition of the multiple stochastic integral. For any $n \geq 0$ we denote by $H^{: n:}:=\mathcal{P}_{n} \ominus \mathcal{P}_{n-1}$ the space of the $n$-th degree Hermite polynomials, see Definition 2.1 of [16]. Here $\mathcal{P}_{-1}:=\{0\}$. It is well known, see Theorem 2.6 of ibid., that $L^{2}(\mathbb{P})=\bigoplus_{n \geq 0} H^{: n}$ :

\section{A variational principle for the resolvent}

We define the random field $\mathbf{V}(\mathbf{x} ; \omega):=\mathbf{v}+\delta \mathbf{F}(\mathbf{x} ; \omega)$ and let $\mathbf{X}(t)$ be the solution of (1.1) with the initial condition $\mathbf{x}$ set at $\mathbf{0}$. The environment process is given by $\eta(t):=\tau_{\mathbf{X}(t)} \omega$. It is an $\Omega_{-}$ valued, deterministic, dynamical system, with $\mathbb{P}$ as its invariant measure. The corresponding group of Koopman operators $P^{t} f(\omega)=f \eta_{t}(\omega), t \geq 0$ extends then to a $C_{0}$-continuous, unitary group on $L^{2}(\mathbb{P})$. Its generator $L$ is given by

$$
L \phi=(\mathbf{v}+\delta \mathbf{F}) \cdot \nabla \phi, \quad \text { for } \phi \in \mathcal{C} .
$$

Here $\nabla \phi:=\left(D_{1} \phi, \ldots, D_{d} \phi\right)$. One can show, in the same way as it was done in see Lemma 4.1 of [19], that $\mathcal{C}$ is invariant under the action of the group $\left(P^{t}\right)$ and, since it is dense in $L^{2}(\mathbb{P})$, it is a core of the generator.

Denote by $R_{\lambda} \phi:=(\lambda-L)^{-1} \phi$ the resolvent operator defined for any $\lambda>0$ and $\phi \in L^{2}(\mathbb{P})$. We can formulate now the variational principle that will be crucial for us in the sequel, cf. Lemma 2.1 of [8].

Proposition 4.1 For any $f \in L^{2}(\mathbb{P})$ and $\lambda>0$ we have

$$
\left\langle R_{\lambda} f, f\right\rangle_{L^{2}(\mathbb{P})}=\sup \left[2\langle f, \phi\rangle_{L^{2}(\mathbb{P})}-\frac{1}{\lambda}\|L \phi\|_{L^{2}(\mathbb{P})}^{2}-\lambda\|\phi\|_{L^{2}(\mathbb{P})}^{2}: \phi \in \mathcal{C}\right] .
$$


Proof. Let $R_{\lambda}^{s}:=(\lambda-L)_{s}^{-1}$ be the symmetric part of the (bounded) operator $R_{\lambda}$ and let $\mathcal{R}$ be the range of $R_{\lambda}^{s}$. As $L$ is anti-selfadjoint, $R_{\lambda}^{s}$ is given explicitly by

$$
R_{\lambda}^{s} f=\frac{1}{2}\left[(\lambda-L)^{-1}+(\lambda+L)^{-1}\right] f=\lambda(\lambda-L)^{-1}(\lambda+L)^{-1} f, \text { for any } f \in L^{2}(\mathbb{P}) .
$$

An elementary calculation shows that for any $f \in L^{2}(\mathbb{P})$ we have, as $\mathcal{R} \in D(L)$,

$$
\begin{aligned}
\left\langle R_{\lambda} f, f\right\rangle_{L^{2}(\mathbb{P})} & =\left\langle R_{\lambda}^{s} f, f\right\rangle_{L^{2}(\mathbb{P})}=\sup \left[2\langle f, \phi\rangle_{L^{2}(\mathbb{P})}-\left\langle\phi,\left(R_{\lambda}^{s}\right)^{-1} \phi\right\rangle_{L^{2}(\mathbb{P})}: \phi \in \mathcal{R}\right] \\
= & \sup \left[2\langle f, \phi\rangle_{L^{2}(\mathbb{P})}-\frac{1}{\lambda}\langle(\lambda-L) \phi,(\lambda-L) \phi\rangle_{L^{2}(\mathbb{P})}: \phi \in \mathcal{R}\right] \\
= & \sup \left[2\langle f, \phi\rangle_{L^{2}(\mathbb{P})}-\lambda\|\phi\|_{L^{2}(\mathbb{P})}^{2}-\frac{1}{\lambda}\|L \phi\|_{L^{2}(\mathbb{P})}^{2}: \phi \in \mathcal{R}\right] .
\end{aligned}
$$

The last equality above uses anti-selfadjointness of $L$. As $\mathcal{C}$ is a core of $L$ we can use it instead of $\mathcal{R}$ as the set of test functions in the variational principles (4.8) - thus, (4.6) follows.

\section{A lower bound on the variance of trajectory fluctuations}

The variational principle (4.6) for the resolvent is used as follows. Let $\mathbf{G}=\left(G_{1}, \ldots, G_{d}\right) \in L^{2}(\mathbb{P})$, the next result concerns the lower bound of the second absolute moment of $\mathbf{z}(t):=\int_{0}^{t} \mathbf{G}(\eta(s)) d s$ (here $\eta(t)$ is the environment process), cf. Lemma 2, p. 655 of [18. To formulate it we introduce

$$
Z_{i}(t):=\int_{0}^{t} \mathbb{E}\left[z_{i}(s)\right]^{2} d s=2 \int_{0}^{t}\left[\int_{0}^{s}\left(\int_{0}^{s_{1}}\left\langle P^{s_{2}} G_{i}, G_{i}\right\rangle_{L^{2}(\mathbb{P})} d s_{2}\right) d s_{1}\right] d s
$$

and $Z(t)=\sum_{i=1}^{d} Z_{i}(t)$. Note that from (4.9) we obtain, in particular that

$$
Z_{i}(t) \leq t^{3}\left\|G_{i}\right\|_{L^{2}(\mathbb{P})}^{2} / 3 .
$$

The following proposition relates the small $\lambda$ behavior of the resolvent to the large time behavior of the process $Z(t)$.

Proposition 4.2 Let $\lambda>0$ and define

$$
G(\lambda):=\sum_{i=1}^{d}\left\langle R_{\lambda} G_{i}, G_{i}\right\rangle_{L^{2}(\mathbb{P})}
$$

Assume that $t, \beta, \rho>0$ are given. Let $T_{\delta}:=t \delta^{-\beta}$. Then, there exist constants $C_{*}, \delta_{0}>0$ such that

$$
Z\left(T_{\delta}^{1+\rho}\right) \geq C_{*} T_{\delta}^{2} G\left(T_{\delta}^{-1}\right), \quad \forall \delta \in\left(0, \delta_{0}\right] .
$$

Proof. Using (4.9) and integration by parts we find that

$$
\begin{aligned}
& \int_{0}^{+\infty} \mathrm{e}^{-\lambda t} Z_{i}(t) d t=2 \iint_{0 \leq s_{2} \leq s_{1} \leq s \leq t} \int_{0} e^{-\lambda t}\left\langle P^{s_{2}} G_{i}, G_{i}\right\rangle_{L^{2}(\mathbb{P})} d s_{1} d s_{2} d s d t \\
& =2 \lambda^{-3} \int_{0}^{+\infty} e^{-\lambda s_{2}}\left\langle P^{s_{2}} G_{i}, G_{i}\right\rangle_{L^{2}(\mathbb{P})} d s_{2}=2 \lambda^{-3}\left\langle R_{\lambda} G_{i}, G_{i}\right\rangle_{L^{2}(\mathbb{P})} .
\end{aligned}
$$


On the other hand, since $t \mapsto Z_{i}(t)$ is an increasing function, we can write that the utmost left hand side of (4.13) is bounded from above as

$$
\begin{aligned}
& \int_{0}^{+\infty} \mathrm{e}^{-\lambda t} Z_{i}(t) d t \leq Z_{i}\left(\lambda^{-(1+\rho)}\right) \int_{0}^{\lambda^{-(1+\rho)}} \mathrm{e}^{-\lambda t} d t+\int_{\lambda^{-(1+\rho)}}^{+\infty} \mathrm{e}^{-\lambda t} Z_{i}(t) d t . \\
& \leq \lambda^{-1} Z_{i}\left(\lambda^{-(1+\rho)}\right)+\frac{1}{3}\left\|G_{i}\right\|_{L^{2}(\mathbb{P})}^{2} \int_{\lambda^{-(1+\rho)}}^{+\infty} \mathrm{e}^{-\lambda t} t^{3} d t \leq \lambda^{-1} Z_{i}\left(\lambda^{-(1+\rho)}\right)+\frac{C\left\|G_{i}\right\|_{L^{2}}^{2}}{3 \lambda^{3}} \int_{\lambda^{-(1+\rho)}}^{+\infty} \mathrm{e}^{-(\lambda t) / 2} d t
\end{aligned}
$$

for some absolute constant $C>0$. We have used (4.10) in the second inequality above. Performing now the integration on the utmost right hand side of (4.14) and recalling (4.13) we obtain that

$$
2 \lambda^{-3}\left\langle R_{\lambda} G_{i}, G_{i}\right\rangle_{L^{2}(\mathbb{P})} \leq \lambda^{-1} Z_{i}\left(\lambda^{-(1+\rho)}\right)+\frac{C \mathrm{e}^{-\lambda^{-\rho}}\left\|G_{i}\right\|_{L^{2}(\mathbb{P})}^{2}}{3 \lambda^{4}} .
$$

Summing up over $i$ we obtain (4.12) upon choosing $\lambda:=T_{\delta}^{-1}$.

\section{The proof of the lower bound}

The lower bound (3.3) in Theorem 3.1 is proved as follows: note that

$$
\mathbf{y}(t)=\delta \int_{0}^{t} \mathbf{F}(\eta(s)) d s
$$

is of the form of the functionals considered in Proposition 4.2 (with $\mathbf{G}=\delta \mathbf{F}$ ). Therefore, to prove (3.3) we will first use suitable test functions in the variational principle (4.6) for the resolvent, and then use (4.12) to obtain the lower bound for $Y(t)$ given by (3.1).

The test functions are chosen as follows: suppose that $\psi(\mathbf{k}) \in C_{0}^{\infty}\left(\mathbb{R}^{d}\right)$ is a smooth complex valued, even vector function: $\psi_{i}(-\mathbf{k})=\psi_{i}^{*}(\mathbf{k}), i=1, \ldots, d$ and denote by $\mathcal{P}_{1}$ the family of all random elements of the form

$$
\phi(\omega)=\int \psi(\mathbf{k}) \cdot \hat{\mathbf{F}}(d \mathbf{k} ; \omega) .
$$

By virtue of (4.6) we have a lower bound

$$
\left\langle R_{\lambda} F_{i}, F_{i}\right\rangle_{L^{2}} \geq \sup \left[2\left\langle F_{i}, \phi\right\rangle_{L^{2}}-\frac{1}{\lambda}\|L \phi\|_{L^{2}}^{2}-\lambda\|\phi\|_{L^{2}}^{2}: \phi \in \mathcal{P}_{1}\right] .
$$

Observe that

$$
2\left\langle F_{i}, \phi\right\rangle_{L^{2}}=2 \int_{\mathbb{R}^{d}} \psi(\mathbf{k}) \cdot \Gamma(\hat{\mathbf{k}}) \mathbf{e}_{i} \frac{a(|\mathbf{k}|) d \mathbf{k}}{|\mathbf{k}|^{2 \alpha+d-2}},
$$

where $\Gamma(\hat{\mathbf{k}})=\left[\Gamma_{i j}(\hat{\mathbf{k}})\right]$. Since we have

$$
D_{j} \phi=i \int_{\mathbb{R}^{d}} k_{j} \psi(\mathbf{k}) \cdot \hat{\mathbf{F}}(d \mathbf{k}),
$$

the operator $L$ acts as

$$
L \phi=(\mathbf{v}+\delta \mathbf{F}) \cdot \nabla \phi=i\left[\int_{\mathbb{R}^{d}} \mathbf{v} \cdot \mathbf{k} \psi(\mathbf{k}) \cdot \hat{\mathbf{F}}(d \mathbf{k})+\delta \int_{\mathbb{R}^{d}} \int_{\mathbb{R}^{d}} \psi(\mathbf{k}) \cdot \hat{\mathbf{F}}(d \mathbf{k}) \mathbf{k} \cdot \hat{\mathbf{F}}\left(d \mathbf{k}^{\prime}\right)\right]
$$


and

$$
\|\phi\|_{L^{2}(\mathbb{P})}^{2}=\int_{\mathbb{R}^{d}}[\Gamma(\hat{\mathbf{k}}) \psi(\mathbf{k}) \cdot \psi(\mathbf{k})] \frac{a(|\mathbf{k}|) d \mathbf{k}}{|\mathbf{k}|^{2 \alpha+d-2}}
$$

we have

$$
\begin{aligned}
& \|L \phi\|_{L^{2}(\mathbb{P})}^{2}=\int_{\mathbb{R}^{d}} \frac{(\mathbf{v} \cdot \mathbf{k})^{2}[\Gamma(\hat{\mathbf{k}}) \psi(\mathbf{k}) \cdot \psi(\mathbf{k})] a(|\mathbf{k}|) d \mathbf{k}}{|\mathbf{k}|^{2 \alpha+d-2}} \\
& +\delta^{2} \int_{\mathbb{R}^{d}} \int_{\mathbb{R}^{d}} \frac{[\Gamma(\hat{\mathbf{k}}) \psi(\mathbf{k}) \cdot \psi(\mathbf{k})]\left[\Gamma\left(\hat{\mathbf{k}}^{\prime}\right) \mathbf{k} \cdot \mathbf{k}\right] a(|\mathbf{k}|) a\left(\left|\mathbf{k}^{\prime}\right|\right) d \mathbf{k} d \mathbf{k}^{\prime}}{\left(|\mathbf{k}|\left|\mathbf{k}^{\prime}\right|\right)^{2 \alpha+d-2}} \\
& +\delta^{2} \int_{\mathbb{R}^{d}} \int_{\mathbb{R}^{d}} \frac{\left[\Gamma(\hat{\mathbf{k}}) \psi(\mathbf{k}) \cdot \mathbf{k}^{\prime}\right]\left[\Gamma\left(\hat{\mathbf{k}}^{\prime}\right) \mathbf{k} \cdot \psi\left(\mathbf{k}^{\prime}\right)\right] a(|\mathbf{k}|) a\left(\left|\mathbf{k}^{\prime}\right|\right) d \mathbf{k} d \mathbf{k}^{\prime}}{\left(|\mathbf{k}|\left|\mathbf{k}^{\prime}\right|\right)^{2 \alpha+d-2}} .
\end{aligned}
$$

Using an elementary estimate

$$
\left(\Gamma(\hat{\mathbf{k}}) \psi(\mathbf{k}) \cdot \mathbf{k}^{\prime}\right) \leq(\Gamma(\hat{\mathbf{k}}) \psi(\mathbf{k}) \cdot \psi(\mathbf{k}))^{1 / 2}\left(\Gamma(\hat{\mathbf{k}}) \mathbf{k}^{\prime} \cdot \mathbf{k}^{\prime}\right)^{1 / 2}
$$

we conclude that

$$
\left\langle R_{\lambda} F_{i}, F_{i}\right\rangle_{L^{2}} \geq \sup \left[\mathfrak{J}_{i}(\phi): \phi \in \mathcal{P}_{1}\right],
$$

where $\mathfrak{J}_{i}(\phi)$ is a quadratic functional given by

$$
\begin{gathered}
\mathfrak{J}_{i}(\phi):=2 \int_{\mathbb{R}^{d}}\left[\psi(\mathbf{k}) \cdot \Gamma(\hat{\mathbf{k}}) \mathbf{e}_{i}\right] \frac{a(|\mathbf{k}|) d \mathbf{k}}{|\mathbf{k}|^{2 \alpha+d-2}}-\lambda \int_{\mathbb{R}^{d}}[\Gamma(\mathbf{k}) \psi(\mathbf{k}) \cdot \psi(\mathbf{k})] \frac{a(|\mathbf{k}|) d \mathbf{k}}{|\mathbf{k}|^{2 \alpha+d-2}} \\
-\frac{1}{\lambda} \int_{\mathbb{R}^{d}} \frac{(\mathbf{v} \cdot \mathbf{k})^{2}[\Gamma(\hat{\mathbf{k}}) \psi(\mathbf{k}) \cdot \psi(\mathbf{k})] a(|\mathbf{k}|) d \mathbf{k}}{|\mathbf{k}|^{2 \alpha+d-2}}-\frac{2 \delta^{2}}{\lambda} \int_{\mathbb{R}^{2 d}} \frac{[\Gamma(\hat{\mathbf{k}}) \psi(\mathbf{k}) \cdot \psi(\mathbf{k})]\left[\Gamma\left(\hat{\mathbf{k}}^{\prime}\right) \mathbf{k} \cdot \mathbf{k}\right] a(|\mathbf{k}|) a\left(\left|\mathbf{k}^{\prime}\right|\right) d \mathbf{k} d \mathbf{k}^{\prime}}{\left(|\mathbf{k}|\left|\mathbf{k}^{\prime}\right|\right)^{2 \alpha+d-2}} .
\end{gathered}
$$

The maximizer of the functional given above equals

$$
\psi_{*}(\mathbf{k})=\Gamma(\hat{\mathbf{k}}) \mathbf{e}_{i}\left\{\lambda+\frac{1}{\lambda}\left[(\mathbf{v} \cdot \mathbf{k})^{2}+\delta^{2}|\mathbf{k}|^{2} \mathcal{H}\right]\right\}^{-1},
$$

where

$$
\mathcal{H}:=2 \int_{\mathbb{R}^{d}} \Gamma\left(\hat{\mathbf{k}}^{\prime}\right) \mathbf{e}_{1} \cdot \mathbf{e}_{1} \frac{a\left(\left|\mathbf{k}^{\prime}\right|\right) d \mathbf{k}^{\prime}}{\left|\mathbf{k}^{\prime}\right|^{2 \alpha+d-2}}=2\left(1-\frac{1}{d}\right) \int_{\mathbb{R}^{d}} \frac{a\left(\left|\mathbf{k}^{\prime}\right|\right) d \mathbf{k}^{\prime}}{\left|\mathbf{k}^{\prime}\right|^{2 \alpha+d-2}}>0 .
$$

Thus, from (4.24) we obtain

$$
P(\lambda):=\sum_{i=1}^{d}\left\langle R_{\lambda} F_{i}, F_{i}\right\rangle_{L^{2}} \geq m(\delta)
$$

where

$$
m(\delta):=(d-1) \int_{\mathbb{R}^{d}}\left\{\lambda+\frac{1}{\lambda}\left[(\mathbf{v} \cdot \mathbf{k})^{2}+\delta^{2}|\mathbf{k}|^{2} \mathcal{H}\right]\right\}^{-1} \frac{a(|\mathbf{k}|) d \mathbf{k}}{|\mathbf{k}|^{2 \alpha+d-2}} .
$$

We claim that $m(\delta) \geq C \lambda^{1-2 \alpha}$ for $0<\lambda \leq 1$. To show this we will assume with no loss of generality that $\mathbf{v}=\mathbf{e}_{1}$. The expression in (4.28) is of the same order of magnitude as

$$
\int_{|\mathbf{k}| \leq 1} \frac{\lambda d \mathbf{k}}{\left(\lambda^{2}+k_{1}^{2}+2 \delta^{2}|\mathbf{k}|^{2} \mathcal{H}\right)|\mathbf{k}|^{2 \alpha+d-2}} \geq C \lambda \int_{|\mathbf{k}| \leq 1} \frac{d \mathbf{k}}{\left(\lambda^{2}+k_{1}^{2}+\delta^{2}|\mathbf{k}|^{2}\right)|\mathbf{k}|^{2 \alpha+d-2}}
$$


for some $C>0$. Writing $\mathbf{k}=\left(k_{1}, \mathbf{l}\right)$ we can further transform the right hand side of (4.29). It equals

$$
C \lambda \int_{k_{1}^{2}+l^{2} \leq 1} \frac{l^{d-2} d k_{1} d l}{\left[\lambda^{2}+\left(1+\delta^{2}\right) k_{1}^{2}+\delta^{2} l^{2}\right]\left(k_{1}^{2}+l^{2}\right)^{\alpha+d / 2-1}},
$$

where $l=|\mathbf{1}|$. Introducing the polar coordinates $l=\varrho \cos \theta, k_{1}=\varrho \sin \theta$ we can estimate (4.30) from below by

$$
C \lambda \int_{0}^{1} \int_{0}^{\pi / 2} \frac{\cos ^{d-2} \theta d \varrho d \theta}{\left(\lambda^{2}+\varrho^{2} \sin ^{2} \theta+\varrho^{2} \delta^{2}\right) \varrho^{2 \alpha-1}} \geq C_{1} \lambda \int_{0}^{1} \int_{0}^{\pi / 4} \frac{d \varrho d \theta}{\left[\lambda^{2}+\varrho^{2}\left(\theta^{2}+\delta^{2}\right)\right] \varrho^{2 \alpha-1}} .
$$

Substituting $\varrho^{\prime}:=\lambda^{-1} \varrho \sqrt{\theta^{2}+\delta^{2}}$ we see that the right hand side of (4.31) equals

$$
C_{1} \lambda^{1-2 \alpha} \int_{0}^{\pi / 4} \frac{d \theta}{\left(\theta^{2}+\delta^{2}\right)^{1-\alpha}}\left[\int_{0}^{\left(\theta^{2}+\delta^{2}\right)^{1 / 2} \lambda^{-1}} \frac{d \varrho}{\left(1+\varrho^{2}\right) \varrho^{2 \alpha-1}}\right]
$$

Since $2-2 \alpha<1$, the first integral above converges even for $\delta=0$, and the second also has a finite limit as $\lambda \rightarrow 0$. We conclude from the above and (4.27) that

$$
P(\lambda) \geq C_{2} \lambda^{1-2 \alpha}, \quad \forall \lambda \in(0,1]
$$

and some $C_{2}>0$.

With the lower bound (4.32) at hand we are ready to finish the proof of Theorem 3.1 Let $\rho^{\prime}>0$ be arbitrary and let $T_{\delta}^{+}$be as in the statement of the theorem. We apply Proposition 4.2 with $T_{\delta}:=\left(T_{\delta}^{+}\right)^{1 /\left(1+\rho^{\prime}\right)}$. Then, for sufficiently small $\delta_{0}$ we have

$$
Y\left(T_{\delta}^{+}\right)=Y\left(T_{\delta}^{1+\rho^{\prime}}\right) \geq C_{3} \delta^{2} T_{\delta}^{2} P\left(T_{\delta}^{-1}\right) \stackrel{(4.32)}{\geq} C_{4} \delta^{2} T_{\delta}^{2 \alpha+1}
$$

for all $\delta \in\left(0, \delta_{0}\right]$ and some $C_{3}, C_{4}>0$. Hence,

$$
\frac{1}{T_{\delta}^{+}} Y\left(T_{\delta}^{+}\right) \geq C_{4} \delta^{2} T_{\delta}^{2 \alpha-\rho^{\prime}}=C_{4} \delta^{2-2 H(1+\rho)\left(2 \alpha-\rho^{\prime}\right) /\left(1+\rho^{\prime}\right)} t^{(1+\rho)\left(2 \alpha-\rho^{\prime}\right) /\left(1+\rho^{\prime}\right)}
$$

and (3.3) follows, provided that $\rho^{\prime}>0$ is chosen sufficiently small.

\section{The upper bound}

The upper bound (3.4) in Theorem 3.1 is proved using an approximation of the trajectory by the correctors.

\section{The corrector fields}

Let $\mathbf{F}_{\lambda}^{(1)}:=\mathbf{F}$ and for any $\lambda>0$ we define the corrector field of the first order in the direction of $\vec{e}_{p}$

$$
\chi_{p, \lambda}^{(1)}:=\int \frac{1}{\lambda-\mathrm{i} \mathbf{v} \cdot \mathbf{k}} \hat{F}_{p}(d \mathbf{k}), \quad p=1, \ldots, d .
$$

Note that $\chi_{p, \lambda}^{(1)}$ satisfies

$$
(\lambda-\mathbf{v} \cdot \nabla) \chi_{p, \lambda}^{(1)}=F_{p, \lambda}^{(1)}, \quad p=1, \ldots, d .
$$


Let us define $\mathbf{F}_{\lambda}^{(2)}=\left(F_{1, \lambda}^{(2)}, \ldots, F_{d, \lambda}^{(2)}\right)$, where

$$
F_{p, \lambda}^{(2)}:=\mathbf{F} \cdot \nabla \chi_{p, \lambda}^{(1)}=i \iint \frac{\mathbf{k}_{1} \cdot \hat{\mathbf{F}}\left(d \mathbf{k}_{2}\right)}{\lambda-\mathrm{i} \mathbf{v} \cdot \mathbf{k}_{1}} \hat{F}_{p}\left(d \mathbf{k}_{1}\right) .
$$

Then, we may write, using the definition of the first order corrector

$$
x_{p}(t)-v_{p} t=\delta \int_{0}^{t} F_{p}(\eta(s)) d s=\delta \lambda \int_{0}^{t} \chi_{p, \lambda}^{(1)}(\eta(s)) d s-\delta \int_{0}^{t} \mathbf{v} \cdot \nabla \chi_{p, \lambda}^{(1)}(\eta(s)) d s .
$$

On the other hand, we also have

$$
\begin{aligned}
& \chi_{p, \lambda}^{(1)}(\eta(t))-\chi_{p, \lambda}^{(1)}(\omega)=\int_{0}^{t}(\mathbf{v}+\delta \mathbf{F}(\eta(s))) \cdot \nabla \chi_{p, \lambda}^{(1)}(\eta(s)) d s \\
& =\int_{0}^{t} \mathbf{v} \cdot \nabla \chi_{p, \lambda}^{(1)}(\eta(s)) d s+\delta \int_{0}^{t} \mathbf{F}^{(2)}(\eta(s)) \cdot \nabla \chi_{p, \lambda}^{(1)}(\eta(s)) d s .
\end{aligned}
$$

so that (4.35) becomes

$$
x_{p}(t)-v_{p} t=\delta^{2} \int_{0}^{t} F_{p, \lambda}^{(2)}(\eta(s)) d s+\delta \lambda \int_{0}^{t} \chi_{p, \lambda}^{(1)}(\eta(s)) d s+\delta \chi_{p, \lambda}^{(1)}(\omega)-\delta \chi_{p, \lambda}^{(1)}(\eta(t))
$$

Now, we may iteratively define $F_{p, \lambda}^{(n)}:=\mathbf{F} \cdot \nabla \chi_{\lambda, p}^{(n-1)}$ and let $\chi_{\lambda, p}^{(n)}$ be the solution of

$$
(\lambda-\mathbf{v} \cdot \nabla) \chi_{\lambda, p}^{(n)}=F_{p, \lambda}^{(n)} .
$$

Then, for any $n \geq 1$ we have a decomposition

$$
x_{p}(t)-v_{p} t=\delta^{n} \int_{0}^{t} F_{p, \lambda}^{(n)}(\eta(s)) d s+\lambda \sum_{l=1}^{n-1} \delta^{l} \int_{0}^{t} \chi_{p, \lambda}^{(l)}(\eta(s)) d s+\sum_{l=1}^{n-1} \delta^{l}\left[\chi_{p, \lambda}^{(l)}(\omega)-\chi_{p, \lambda}^{(l)}(\eta(t))\right] .
$$

Technically, the most important results of this section are the following bounds for the correctors.

Proposition 4.3 Suppose that $\alpha \in(1 / 2,1)$. Then, for each $n \geq 1$ we have

$$
\left\|\chi_{\lambda}^{(n)}\right\|_{L^{2}} \leq \frac{C}{\lambda^{n \alpha}}(1+|\log \lambda|)^{n / 2}, \quad \lambda \in(0,1]
$$

for some constant $C>0$. When, on the other hand $\alpha<1 / 2$ we have

$$
\left\|\chi_{\lambda}^{(n)}\right\|_{L^{2}} \leq \frac{C}{\lambda^{n / 2}}(1+|\log \lambda|)^{n / 2}, \quad \lambda \in(0,1] .
$$

As a consequence we obtain the following.

Corollary 4.4 For some constant $C>0$ we have

$$
\left\|\mathbf{F}_{\lambda}^{(n)}\right\|_{L^{2}} \leq \frac{C}{\lambda^{n \alpha}}(1+|\log \lambda|)^{n / 2}, \quad \lambda \in(0,1]
$$

for $\alpha \in(1 / 2,1)$ and

$$
\left\|\mathbf{F}_{\lambda}^{(n)}\right\|_{L^{2}} \leq \frac{C}{\lambda^{n / 2}}(1+|\log \lambda|)^{n / 2}, \quad \lambda \in(0,1]
$$

for $\alpha<1 / 2$. 
The proof of Proposition 4.3 is rather technical and we postpone it until Section 6, where the proof of Corollary 4.4 can also be found.

In what follows we shall also need the following lemma which shows that the inner products of correctors with $\mathbf{F}$ are of a smaller order than one would naively expect from the Cauchy-Schwartz inequality and Proposition 4.3

Lemma 4.5 Suppose that $\alpha<1 / 2$. Then, for a given $n \geq 1$ there exists a constant $C>0$ such that

$$
\left|\left\langle\chi_{\lambda, p}^{(n)}, F_{p}\right\rangle_{L^{2}}\right| \leq \frac{C}{\lambda^{(n-1) / 2}}(1+|\log \lambda|)^{(n+1) / 2}, \quad \forall \lambda \in(0,1] .
$$

This lemma is also proved in Section 6 ,

\section{The proof of the upper bound}

We now prove (3.4). Using (4.39) we estimate

$$
\mathbb{E}\left|\mathbf{y}\left(T_{\delta}^{-}\right)\right|^{2} \leq C\left[\delta^{2 n}\left(T_{\delta}^{-}\right)^{2}\left\|\mathbf{F}_{\lambda}^{(n)}\right\|_{L^{2}}^{2}+\sum_{l=1}^{n-1} \delta^{2 l}\left(\lambda T_{\delta}^{-}\right)^{2} \sum_{p=1}^{d}\left\|\chi_{p, \lambda}^{(l)}\right\|_{L^{2}}^{2}+\sum_{l=1}^{n-1} \delta^{2 l} \sum_{p=1}^{d}\left\|\chi_{p, \lambda}^{(l)}\right\|_{L^{2}}^{2}\right]
$$

Recall that $T_{\delta}^{-}=\delta^{-2 H(1-\rho)} t^{1-\rho}$. This together with estimates (4.40) and (4.42) imply that the right hand side of (4.45) can be estimated by

$$
C\left[\left(\delta \lambda^{-\alpha}\right)^{2 n}\left(T_{\delta}^{-}\right)^{2}+\sum_{l=1}^{n-1}\left(\delta \lambda^{-\alpha}\right)^{2 l}\left(\lambda T_{\delta}^{-}\right)^{2}+\sum_{l=1}^{n-1}\left(\delta \lambda^{-\alpha}\right)^{2 l}\right] .
$$

Choose $\lambda:=\delta^{2 H(1-\rho)}$, we obtain then

$$
\mathbb{E}\left|\mathbf{y}\left(T_{\delta}^{-}\right)\right|^{2} \leq C t^{2(1-\rho)}\left(\delta^{n \rho} \delta^{-4 H(1-\rho)}+\sum_{l=1}^{n-1} \delta^{l \rho}\right) .
$$

For an arbitrary $\rho>0$ we can choose $n$ sufficiently large so that the right hand side of (4.46) is of order of magnitude $o(1)$ so that (3.4) follows.

\section{The proof of Theorem 3.2}

For the most part the proof of the upper bound (3.6) in Theorem 3.2 is a repetition of what has been done in the corresponding situation in the previous case. Observe that (4.45) still holds with $T_{\delta}^{-}:=t \delta^{-2(1-\rho)}$. We can choose now $\lambda:=\left(T_{\delta}^{-}\right)^{-1}$ and easily convince ourselves that (3.6) holds.

The heuristic reason why this direction is simpler once the upper bound in Theorem 3.1 has been obtained is that the time $\delta^{-2}$ is much shorter that $\delta^{-2 H}, H=1 /(2 \alpha)$ for $\alpha<1 / 2$. Therefore the fact that "nothing happens until the time $O\left(\delta^{-2}\right)$ " is not very surprising in Theorem 3.2. The lower bound in Theorem 3.2 is more informative - it tells that something happens at the time $O\left(\delta^{-2}\right)$ which is much earlier than the time scale $\delta^{-2 H}$.

We now prove the lower bound (3.5). Let $\rho^{\prime}>0$ and $T_{\delta}:=\left(t \delta^{-2}\right)^{1 /\left(1+\rho^{\prime}\right)}$. We shall further specify the parameter $\rho^{\prime}$ later on. By virtue of Proposition 4.2 we conclude that for a certain $C>0$ and sufficiently small $\delta>0$

$$
Y\left(T_{\delta}^{1+\rho^{\prime}}\right) \geq C \delta^{2} T_{\delta}^{2} P\left(T_{\delta}^{-1}\right)
$$


where $P(\lambda)$ is given by (4.27). Recall that the correctors $\chi_{\lambda, p}^{(n)}$ are the solutions of (4.38) for an arbitrary $\lambda>0$ and $N \geq n \geq 1$. Let the remainders $r_{\lambda, p}^{(N)}$ be given by

$$
[\lambda-(\mathbf{v}+\delta \mathbf{F}) \cdot \nabla] r_{\lambda, p}^{(N)}=F_{p, \lambda}^{(N+1)}
$$

Multiplying both sides of (5.2) by $r_{\lambda, p}^{(N)}$ and integrating out we get an obvious bound

$$
\left\|r_{\lambda, p}^{(N)}\right\|_{L^{2}} \leq \frac{1}{\lambda}\left\|F_{p, \lambda}^{(N+1)}\right\|_{L^{2}} \stackrel{\text { (4.431) }}{\leq} \frac{C}{\lambda^{1+(N+1) / 2}}(1+|\log \lambda|)^{N / 2}
$$

Observe also that

$$
R_{\lambda} F_{p}=\sum_{n=1}^{N} \delta^{n-1} \chi_{\lambda, p}^{(n)}+\delta^{N} r_{\lambda, p}^{(N)}
$$

and therefore

$$
\left\langle R_{\lambda} F_{p}, F_{p}\right\rangle_{L^{2}} \geq\left\langle\chi_{\lambda, p}^{(1)}, F_{p}\right\rangle_{L^{2}}-C \sum_{n=1}^{N-1} \delta^{n}\left|\left\langle\chi_{\lambda, p}^{(n+1)}, F_{p}\right\rangle_{L^{2}}\right|-C \lambda^{-3 / 2}\left(\delta \lambda^{-1 / 2}\right)^{N}(1+|\log \lambda|)^{N / 2} .
$$

Lemma 4.5 allows us to obtain the following estimate

$$
\left\langle R_{\lambda} F_{p}, F_{p}\right\rangle_{L^{2}} \geq\left\langle\chi_{\lambda, p}^{(1)}, F_{p}\right\rangle_{L^{2}}-C \sum_{n=1}^{N-1}\left(\delta \lambda^{-1 / 2}\right)^{n} \mid(1+|\log \lambda|)^{n / 2}-C \lambda^{-3 / 2}\left(\delta \lambda^{-1 / 2}\right)^{N}(1+|\log \lambda|)^{N / 2} .
$$

Choosing $\lambda:=T_{\delta}^{-1}$ we obtain from (5.1) and (5.4), with sufficiently large $N$ chosen, that

$$
\delta^{2-\rho} Y\left(t \delta^{-2}\right) \geq C t^{2 /\left(1+\rho^{\prime}\right)} \delta^{-\rho+4 \rho^{\prime} /\left(1+\rho^{\prime}\right)},
$$

which clearly implies (3.5), provided that $-\rho+4 \rho^{\prime} /\left(1+\rho^{\prime}\right)<0$.

\section{The proofs of the corrector bounds}

In this section we prove the technical bounds on the correctors stated in Proposition 4.3. Corollary 4.4 and Lemma 4.5.

\section{The Feynman diagrams}

The proofs of the corrector bounds make an extensive use of the Feynman diagrams. Let us recall now the corresponding basic notions. Let $Z_{n}:=\{1, \ldots, n\}$, a Feynman diagram $\mathcal{F}$ (of order $n \geq 0$ and rank $r \geq 0$ ) based on $Z_{n}$ is a graph consisting of a set $B(\mathcal{F}) \subset Z_{n}$ of vertices from $Z_{n}$, and a set $E(\mathcal{F})$ of $e(\mathcal{F})$ edges connecting points in $Z_{n}$ without common endpoints. So there are $e(\mathcal{F})$ pairs of vertices, each joined by an edge, and $a(\mathcal{F}):=n-2 e(\mathcal{F})$ unpaired vertices, called free vertices. An edge whose endpoints are $m, n \in B$ is denoted by $\widehat{m n}$ (unless otherwise specified, we always assume $m<n)$. A diagram $\mathcal{F}$ is said to be based on $B(\mathcal{F})$. Denote the set of free vertices by $A(\mathcal{F})$, so $A(\mathcal{F})=\mathcal{F} \backslash E(\mathcal{F})$. The diagram is complete if $A(\mathcal{F})$ is empty and incomplete, otherwise. Also for a given $1 \leq p \leq n$ let us denote by $A_{p}(\mathcal{F})$ the set of all free vertices that are less or equal to $p$ and by $L_{p}(\mathcal{F})$ the union of $A_{p}(\mathcal{F})$ and all the left vertices of edges $\widehat{m n}$ for which $m \leq p<n$. Let $L(\mathcal{F}):=L_{n}(\mathcal{F})$ denote the left vertices of all edges belonging to $E(\mathcal{F})$. 


\section{Expressions for correctors}

We begin with some explicit expressions for the correctors $\chi_{m, \lambda}^{(n)}$. Recall that the functions $\chi_{m, \lambda}^{(n)}$, $m=1,2 \ldots, d$, are defined iteratively as the solutions of

$$
(\lambda-\mathbf{v} \cdot \nabla) \chi_{m, \lambda}^{(n)}=F_{m, \lambda}^{(n)}, \quad F_{m, \lambda}^{(n)}:=\mathbf{F} \cdot \nabla \chi_{m, \lambda}^{(n-1)}
$$

with $\chi_{m, \lambda}^{(1)}$, the solution of

$$
(\lambda-\mathbf{v} \cdot \nabla) \chi_{m, \lambda}^{(1)}=F_{m, \lambda}, \quad m=1, \ldots, d
$$

given explicitly by

$$
\chi_{m, \lambda}^{(1)}:=\int \frac{1}{\lambda-\mathrm{i} \mathbf{v} \cdot \mathbf{k}} \hat{F}_{m}(d \mathbf{k}), \quad m=1, \ldots, d .
$$

Let us introduce auxiliary functions

$$
\tilde{h}_{n}\left(\mathbf{k}_{1}, \ldots, \mathbf{k}_{n}\right):=\frac{\sum_{l=1}^{n} \mathbf{k}_{l}}{\lambda-\mathrm{iv} \cdot\left(\sum_{l=1}^{n} \mathbf{k}_{l}\right)}
$$

for $n \geq 1$ and

$$
h_{1}^{(m)}\left(\mathbf{k}_{1}\right):=\frac{\mathbf{e}_{m}}{\lambda-\mathrm{i} \mathbf{v} \cdot \mathbf{k}_{1}}, \quad h_{n}\left(\mathbf{k}_{1}, \ldots, \mathbf{k}_{n}\right):=\frac{\sum_{l=1}^{n-1} \mathbf{k}_{l}}{\lambda-\mathrm{i} \mathbf{v} \cdot\left(\sum_{l=1}^{n} \mathbf{k}_{l}\right)}, \quad n \geq 2 .
$$

Then, a simple induction argument shows that the $n$-th order corrector in the direction of the vector $\mathbf{e}_{p}$ is given by

$$
\chi_{m, \lambda}^{(n)}:=i^{n-1} \int h_{1}^{(m)}\left(\mathbf{k}_{1}\right) \otimes h_{2}\left(\mathbf{k}_{1}, \mathbf{k}_{2}\right) \otimes \ldots \otimes h_{n}\left(\mathbf{k}_{1}, \ldots, \mathbf{k}_{n}\right) \cdot \hat{\mathbf{F}}\left(d \mathbf{k}_{1}\right) \otimes \hat{\mathbf{F}}\left(d \mathbf{k}_{2}\right) \ldots \otimes \hat{\mathbf{F}}\left(d \mathbf{k}_{n}\right)
$$

and the fields $\mathbf{F}_{\lambda}^{(n)}$ defined in (6.1) are $\mathbf{F}_{\lambda}^{(n)}=\left(F_{1, \lambda}^{(n)}, \ldots, F_{d, \lambda}^{(n)}\right)$, where

$$
F_{m, \lambda}^{(n)}:=i^{n-2} \int \mathbf{e}_{m} \otimes \tilde{h}_{1}\left(\mathbf{k}_{1}\right) \otimes \ldots \otimes \tilde{h}_{n-1}\left(\mathbf{k}_{1}, \ldots, \mathbf{k}_{n-1}\right) \cdot \hat{\mathbf{F}}\left(d \mathbf{k}_{1}\right) \otimes \hat{\mathbf{F}}\left(d \mathbf{k}_{2}\right) \ldots \otimes \hat{\mathbf{F}}\left(d \mathbf{k}_{n}\right) .
$$

\section{The proof of Proposition 4.3}

\section{The Feynman diagrams expansion}

We now prove Proposition 4.3 an $L^{2}$-bound for the correctors. Using the Feynman diagram expansion (A.7) of a multiple stochastic integral from the Appendix we can write that

$$
\chi_{\lambda}^{(n)}=\sum_{\mathcal{F}} \chi_{\lambda}^{(n)}(\mathcal{F})
$$

where the summation extends over all Feynman diagrams $\mathcal{F}$ of the set $Z_{n}$. For a given diagram $\mathcal{F}$ with free vertices $A(\mathcal{F})=\left\{n_{1}, \ldots, n_{a}\right\}$ and edges $E(\mathcal{F})=\{\widehat{p q}\}$ we set

$$
\chi_{m, \lambda}^{(n)}(\mathcal{F}):=: \int \ldots \int f^{m, \lambda}\left(\mathbf{k}_{n_{1}}, \ldots, \mathbf{k}_{n_{a}}\right) \cdot \hat{\mathbf{F}}\left(d \mathbf{k}_{n_{1}}\right) \otimes \ldots \otimes \hat{\mathbf{F}}\left(d \mathbf{k}_{n_{a}}\right):
$$


where : $:$ denotes the orthogonal projection onto the space of $a$-th degree Hermite polynomials $H^{: a}$, cf Definition 3.1 of [16], and $f^{m, \lambda}:\left(\mathbb{R}^{d}\right)^{a} \rightarrow\left(\mathbb{R}^{d}\right)^{a}$ is given by

$$
f_{i_{n_{1}, \ldots, i_{n_{a}}}^{m, \lambda}}^{m}\left(\mathbf{k}_{n_{1}}, \ldots, \mathbf{k}_{n_{a}}\right):=\int h_{1, i_{1}}^{(m)}\left(\mathbf{k}_{1}\right) \ldots h_{n, i_{n}}\left(\mathbf{k}_{1}, \ldots, \mathbf{k}_{n}\right) \prod_{\widehat{p} q \in E(\mathcal{F})} \Gamma_{i_{p}, i_{q}}\left(\hat{\mathbf{k}}_{p}\right) \frac{a\left(\left|\mathbf{k}_{p}\right|\right) \delta\left(\mathbf{k}_{p}+\mathbf{k}_{q}\right)}{\left|\mathbf{k}_{p}\right|^{2 \alpha+d-2}} d \mathbf{k}_{p} d \mathbf{k}_{q} .
$$

Using Theorem 3.9 p. 26 of [16] one concludes that

$$
\begin{aligned}
\left\|\chi_{m, \lambda}^{(n)}(\mathcal{F})\right\|_{L^{2}}^{2} & =\sum_{\pi} \int f^{m, \lambda}\left(\mathbf{k}_{n_{1}}, \ldots, \mathbf{k}_{n_{a}}\right) f^{m, \lambda}\left(\mathbf{k}_{\pi\left(n_{1}\right)}^{\prime}, \ldots, \mathbf{k}_{\pi\left(n_{a}\right)}^{\prime}\right) \prod_{p=1}^{a} \Gamma_{n_{p}, n_{\pi(p)}}\left(\hat{\mathbf{k}}_{n_{p}}\right) \frac{a\left(\left|\mathbf{k}_{n_{p}}\right|\right)}{\left|\mathbf{k}_{n_{p}}\right|^{2 \alpha+d-2}} \\
& \times \delta\left(\mathbf{k}_{n_{p}}+\mathbf{k}_{\pi\left(n_{p}\right)}^{\prime}\right) d \mathbf{k}_{n_{p}} d \mathbf{k}_{\pi\left(n_{p}\right)}^{\prime},
\end{aligned}
$$

where the summation extends over all permutations $\pi: A(\mathcal{F}) \rightarrow A(\mathcal{F})$. We assume with no loss of

generality that $\mathbf{v}=\mathbf{e}_{1}$. Also we write $\mathbf{k}_{i}=\left(k_{i}, \mathbf{l}_{i}\right) \in \mathbb{R} \times \mathbb{R}^{d-1}$, splitting out the first component of the vector $\mathbf{k}_{i}$. Then, with this notation we have

$$
\begin{aligned}
\left|f_{i_{n_{1}}, \ldots, i_{n_{a}}}^{m, \lambda}\left(\mathbf{k}_{n_{1}}, \ldots, \mathbf{k}_{n_{a}}\right)\right| \leq & \int_{-K}^{K} \ldots \int_{-K}^{K} \prod_{j=1}^{n} \frac{1}{\left|\lambda-\mathrm{i} \sum_{l=1}^{j} k_{l}\right|} \prod_{\widehat{p} q \in E(\mathcal{F})} \frac{\delta\left(k_{p}+k_{q}\right)}{\left|k_{p}\right|^{2 \alpha-1}} d k_{p} d k_{q} \\
& \times\left\{\int \ldots \prod_{\left|\mathbf{l}_{p}\right| \leq K} \prod_{\hat{p} q \in E(\mathcal{F})} \frac{\delta\left(\mathbf{l}_{p}+\mathbf{l}_{q}\right) d \mathbf{l}_{p} d \mathbf{l}_{q}}{\left(k_{p}^{2}+\left|\mathbf{l}_{p}\right|^{2}\right)^{(d-1) / 2}}\right\} .
\end{aligned}
$$

The last integral can be estimated by $C \prod_{\widehat{p} q \in E(\mathcal{F})}\left(1+\log ^{+}\left|k_{p}\right|\right)$ so we conclude that the right hand side of (6.6) can be estimated by

$$
\begin{aligned}
& a(\mathcal{F}) ! \int\left|f^{m, \lambda}\right|^{2}\left(\mathbf{k}_{n_{1}}, \ldots, \mathbf{k}_{n_{a}}\right) \prod_{p=1}^{a} \frac{a\left(\left|\mathbf{k}_{n_{p}}\right|\right) d \mathbf{k}_{n_{p}}}{\left|\mathbf{k}_{n_{p}}\right|^{2 \alpha+d-2}} \\
\leq & C \int_{-K}^{K} \ldots \int_{-K}^{K} \tilde{f}_{\lambda}^{2}\left(k_{n_{1}}, \ldots, k_{n_{a}}\right) \prod_{p=1}^{a} \frac{\left(1+\log ^{+}\left|k_{n_{p}}\right|\right) d k_{n_{p}}}{\left|k_{n_{p}}\right|^{2 \alpha-1}}
\end{aligned}
$$

where

$$
\tilde{f}_{\lambda}\left(k_{n_{1}}, \ldots, k_{n_{a}}\right):=\int_{-K}^{K} \ldots \int_{-K}^{K} \prod_{j=1}^{n} \frac{1}{\left|\lambda-\mathrm{i} \sum_{l=1}^{j} k_{l}\right|} \prod_{\widehat{p} q \in E(\mathcal{F})} \frac{\left(1+\log ^{+}\left|k_{p}\right|\right) \delta\left(k_{p}+k_{q}\right)}{\left|k_{p}\right|^{2 \alpha-1}} d k_{p} d k_{q} .
$$

\section{The proof of (4.40)}

Suppose first that $\alpha \in(1 / 2,1)$ - we need to prove the estimate (4.40) from Proposition 4.3 Note that after changing variables $k_{j}=\lambda k_{j}^{\prime}$ and dropping the primes we get the estimate of the left hand side of (6.7) as

$$
\left\|\chi_{m, \lambda}^{(n)}(\mathcal{F})\right\|_{L^{2}}^{2} \leq C \lambda^{-2 \alpha n}(1+|\log \lambda|)^{n} I,
$$


where

$$
I:=\int_{-\infty}^{\infty} \ldots \int_{-\infty}^{\infty} g^{2}\left(k_{n_{1}}, \ldots, k_{n_{a}}\right) \prod_{p=1}^{a} \frac{\left(1+\log ^{+}\left|k_{n_{p}}\right|\right) d k_{n_{p}}}{\left|k_{n_{p}}\right|^{2 \alpha-1}}
$$

with

$$
g\left(k_{n_{1}}, \ldots, k_{n_{a}}\right):=\int_{-\infty}^{\infty} \ldots \int_{-\infty}^{\infty} \prod_{j=1}^{n} \frac{1}{1+\left|\sum_{l=1}^{j} k_{l}\right|} \prod_{\widehat{p q} \in E(\mathcal{F})} \frac{\left(1+\log ^{+}\left|k_{p}\right|\right) \delta\left(k_{p}+k_{q}\right)}{\left|k_{p}\right|^{2 \alpha-1}} d k_{p} d k_{q}
$$

We shall show by an elementary calculation that $I<+\infty$ - this is all that remains to prove the estimate (4.40). We present the details for the convenience of the reader: note that

$$
\begin{aligned}
I= & \int \ldots \prod_{\mathbb{R}^{a+4 e}} \prod_{j=1}^{n} \frac{1}{1+\left|\sum_{l=1}^{j} k_{l}\right|} \times \prod_{j^{\prime}=1}^{n} \frac{1}{1+\left|\sum_{l^{\prime}=1}^{j^{\prime}} k_{l^{\prime}}^{\prime}\right|} \times \prod_{m=1}^{a} \frac{\left(1+\log ^{+}\left|k_{n_{m}}\right|\right) d k_{n_{m}}}{\left|k_{n_{m}}\right|^{2 \alpha-1}} \\
& \times \prod_{\widehat{p} q \in E(\mathcal{F})} \frac{\left(1+\log ^{+}\left|k_{p}\right|\right) \delta\left(k_{p}+k_{q}\right) d k_{p} d k_{q}}{\left|k_{p}\right|^{2 \alpha-1}} \times \prod_{p^{\prime} q^{\prime} \in E(\mathcal{F})} \frac{\left(1+\log ^{+}\left|k_{p^{\prime}}\right|\right) \delta\left(k_{p^{\prime}}+k_{q^{\prime}}\right) d k_{p^{\prime}}^{\prime} d k_{q^{\prime}}}{\left|k_{p^{\prime}}\right|^{2 \alpha-1}} \\
& =\int_{\mathbb{R}^{n}} \prod_{j=1}^{n} \frac{1}{1+\left|\sum_{l=1}^{j} k_{l}\right|} \times \prod_{j^{\prime}=1}^{n} \frac{1}{1+\left|\sum_{l^{\prime}=1}^{j^{\prime}} k_{l^{\prime}}^{\prime}\right|} \times \prod_{m=1}^{a} \frac{\left(1+\log ^{+}\left|k_{n_{m}}\right|\right) d k_{n_{m}}}{\left|k_{n_{m}}\right|^{2 \alpha-1}} \\
& \times \prod_{p \in L(\mathcal{F})} \frac{\left(1+\log ^{+}\left|k_{p}\right|\right) d k_{p}}{\left|k_{p}\right|^{2 \alpha-1}} \times \prod_{p^{\prime} \in L(\mathcal{F})} \frac{\left(1+\log ^{+}\left|k_{p^{\prime}}\right|\right) d k_{p^{\prime}}^{\prime}}{\left|k_{p^{\prime}}\right|^{2 \alpha-1}} .
\end{aligned}
$$

We adopt the rule that in the sums above $k_{r}+k_{s}=0$ and $k_{r}^{\prime}+k_{s}^{\prime}=0$, if $\widehat{r s}$ is an edge. Now, the integration over $|k| \leq 1$ is not a problem so in order to show that $I<+\infty$ it suffices only to prove that

$$
\begin{aligned}
& I^{\prime}=\int_{\mathbb{R}^{n}} \prod_{j=1}^{n} \frac{1}{1+\left|\sum_{l=1}^{j} k_{l}\right|} \times \prod_{j^{\prime}=1}^{n} \frac{1}{1+\left|\sum_{l^{\prime}=1}^{j^{\prime}} k_{l^{\prime}}^{\prime}\right|} \\
& \times \prod_{m=1}^{a} \frac{\left(1+\log ^{+}\left|k_{n_{m}}\right|\right) d k_{n_{m}}}{1+\left|k_{n_{m}}\right|^{2 \alpha-1}} \times \prod_{p \in L(\mathcal{F})} \frac{\left(1+\log ^{+}\left|k_{p}\right|\right) d k_{p}}{1+\left|k_{p}\right|^{2 \alpha-1}} \times \prod_{p^{\prime} \in L(\mathcal{F})} \frac{\left(1+\log ^{+}\left|k_{p^{\prime}}\right|\right) d k_{p^{\prime}}^{\prime}}{1+\left|k_{p^{\prime}}\right|^{2 \alpha-1}}<+\infty .
\end{aligned}
$$

Using Hölder inequality with $\bar{p}$ such that $\bar{p}(2 \alpha-1)=2$ and $q=2 /(3-2 \alpha)>1$ such that $1 / \bar{p}+1 / q=1$ leads to $I^{\prime} \leq C(I I)^{1 / q}$, with

$$
I I=\int_{\mathbb{R}^{n}} \prod_{p=1}^{n} \frac{1}{1+\left|\sum_{l=1}^{p} k_{l}\right|^{q}} \times \prod_{p^{\prime}=1}^{n} \frac{1}{1+\left|\sum_{l^{\prime}=1}^{p^{\prime}} k_{l^{\prime}}^{\prime}\right|^{q}} \times \prod_{r \in A(\mathcal{F})} d k_{r} \times \prod_{m \in L(\mathcal{F})} d k_{m} \times \prod_{r^{\prime} \in L(\mathcal{F})} d k_{r^{\prime}}^{\prime} .
$$

Now, we introduce new variables $\eta_{1}, \ldots, \eta_{n}$ as follows: first, we look at the sums $S_{m}=\sum_{j=1}^{m} k_{j}$ with $m=1, \ldots, n$, with the terms $k_{r}+k_{s}=0$ if $\widehat{r s}$ is an edge. We say that $\eta_{1}=k_{1}$, then we take $j_{2}$ that is the smallest number larger than one which is either free or a left vertex, and let $\eta_{2}=S_{j_{2}}$. Note that either $\eta_{2}=k_{2}$ if $k_{2}$ is not a right end, or $\eta_{2}=k_{3}$ if $\left(k_{1} k_{2}\right)$ is an edge. We continue in the same way: having defined $\eta_{l-1}=S_{j_{l-1}}$ we take $j_{l}$ to be the first index larger than $j_{l-1}$ which is not a right end of an edge, and take $\eta_{l}=S_{j_{l}}$. In this way we will pick $n-e(\mathcal{F})=a(\mathcal{F})+e(\mathcal{F})$ sums out of $S_{m}, m=1, \ldots, n$ and will define $\eta_{1}, \eta_{2}, \ldots, \eta_{n-e(\mathcal{F})}$. Note that the change of variables is lower-triangular. Then, we do a similar procedure with the $k^{\prime}$-variables - we look at $P_{m}=\sum_{j=1}^{m} k_{j}^{\prime}$ 
and set $\eta_{n-e+l}=P_{q_{l}}, l=1, \ldots, e$, where $q_{1}<\ldots<q_{e}$ are the left vertices. This defines all $\eta_{l}$, $l=1, \ldots, n$ and keeps the change of variables lower-triangular. Hence, it is invertible with the Jacobian equal to 1 . As a consequence, we may drop all sums in the denominator which are not equal to one of $\eta_{j}$ and we have

$$
I I \leq \int_{\mathbb{R}^{n}} \prod_{j=1}^{n} \frac{1}{1+\left|\eta_{j}\right|^{q}} d \eta_{j}<+\infty .
$$

It follows that $I<+\infty$ and the proof of (4.40) is complete.

\section{The proof of (4.41)}

It remains to prove the estimate (4.41) in Proposition 4.3. Suppose now that $\alpha<1 / 2$. The right hand side of (6.6) can be estimated by

$$
I:=C \int_{-K}^{K} \ldots \int_{-K}^{K} \tilde{f}_{\lambda}^{2}\left(k_{n_{1}}, \ldots, k_{n_{a}}\right) \prod_{p=1}^{a} d k_{n_{p}}
$$

where

$$
\tilde{f}_{\lambda}\left(k_{n_{1}}, \ldots, k_{n_{a}}\right):=\int_{-K}^{K} \ldots \int_{-K}^{K} \prod_{j=1}^{n} \frac{1}{\left|\lambda-i \sum_{l=1}^{j} k_{l}\right|} \prod_{\widehat{p} q \in E(\mathcal{F})} \delta\left(k_{p}+k_{q}\right) d k_{p} d k_{q}
$$

We have

$$
\begin{aligned}
& I \leq C \int_{-K}^{K} \ldots \int_{-K}^{K} \prod_{j=1}^{n} \frac{1}{\lambda+\left|\sum_{l=1}^{j} k_{l}\right|} \times \prod_{j^{\prime}=1}^{n} \frac{1}{\lambda+\left|\sum_{l^{\prime}=1}^{j^{\prime}} k_{l^{\prime}}^{\prime}\right|} \\
& \times \prod_{m=1}^{a} d k_{n_{m}} \times \prod_{\widehat{p q} \in E(\mathcal{F})} \delta\left(k_{p}+k_{q}\right) d k_{p} d k_{q} \times \prod_{\widehat{p^{\prime} q^{\prime}} \in E(\mathcal{F})} \delta\left(k_{p^{\prime}}+k_{q^{\prime}}\right) d k_{p^{\prime}}^{\prime} d k_{q^{\prime}} .
\end{aligned}
$$

We introduce the new variables $\eta_{1}, \ldots, \eta_{n}$ as in the previous case. With the help of this transformation and replacing the remaining $n$ of the denominators above by $1 / \lambda$ we conclude that

$$
\left\|\chi_{\lambda}^{(n)}(\mathcal{F})\right\|_{L^{2}}^{2} \leq \frac{C}{\lambda^{n}} \int_{-n K}^{n K} \ldots \int_{-n K}^{n K} \prod_{j=1}^{n} \frac{1}{\lambda+\left|\eta_{j}\right|} d \eta_{j} \leq \frac{C}{\lambda^{n}}\left[\log \left(\frac{1}{\lambda}\right)+1\right]^{n}
$$

for some $C>0$ and all $\lambda \in(0,1]$. Thus (4.41) follows.

\section{The proof of Corollary 4.4}

We now prove the estimate (4.43) in Corollary 4.4 Using the Cauchy-Schwartz inequality we obtain

$$
\left\|\mathbf{F}_{\lambda}^{(n)}\right\|_{L^{2}} \leq\|\mathbf{F}\|_{L^{4}}\left\|\nabla \chi_{\lambda}^{(n)}\right\|_{L^{4}}
$$

However, since all the $L^{p}$ norms, $1 \leq p<+\infty$ on $\mathcal{P}_{n}$ are equivalent, see Theorem 5.10 p. 62 of [16], and $\mathbf{F} \in \mathcal{P}_{1}, \nabla \chi_{\lambda}^{(n)} \in \mathcal{P}_{n}$ we conclude that

$$
\left\|\mathbf{F}_{\lambda}^{(n)}\right\|_{L^{2}} \leq C\|\mathbf{F}\|_{L^{2}}\left\|\nabla \chi_{\lambda}^{(n)}\right\|_{L^{2}}
$$

for some constant $C>0$. Thanks to the fact that the spectral measure $\hat{\mathbf{F}}$ has a compact support the gradient operator $\nabla$ restricted to $\mathcal{P}_{n}$ is bounded (this can be seen by a direct calculation $D_{p}$ on elements of the form (4.5)). This in turn implies (4.43). 


\section{The proof of Lemma 4.5}

Finally, we prove the estimate (4.44) in Lemma 4.5. We only need to be concerned with $n$ odd, for otherwise the left hand side of (4.44) vanishes. Using expansion (6.5) we can write that

$$
\left\langle\chi_{\lambda, p}^{(n)}, F_{p}\right\rangle_{L^{2}}=\sum_{\mathcal{F}}\left\langle\chi_{\lambda, p}^{(n)}(\mathcal{F}), F_{p}\right\rangle_{L^{2}}
$$

and the summation extends over those Feynman diagrams $\mathcal{F}$ for which $a(\mathcal{F})=1$. Suppose that $q$ is the only free vertex of $\mathcal{F}$. Then,

$$
\begin{aligned}
\left\langle\chi_{\lambda, p}^{(n)}(\mathcal{F}), F_{p}\right\rangle_{L^{2}} & =\sum_{i_{1}, \ldots, i_{n}} \int \ldots \int h_{1, i_{1}}^{(p)}\left(\mathbf{k}_{1}\right) \ldots h_{n, i_{n}}\left(\mathbf{k}_{1}, \ldots, \mathbf{k}_{n}\right) \Gamma_{i_{q}, p}\left(\hat{\mathbf{k}}_{q}\right) \\
& \times \frac{a\left(\left|\mathbf{k}_{q}\right|\right)}{\left|\mathbf{k}_{q}\right|^{2 \alpha+d-2}} \delta\left(\mathbf{k}_{q}+\mathbf{k}_{n+1}\right) d \mathbf{k}_{q} d \mathbf{k}_{n+1} \prod_{\hat{r s} \in E(\mathcal{F})} \Gamma_{i_{r}, i_{s}}\left(\hat{\mathbf{k}}_{r}\right) \frac{a\left(\left|\mathbf{k}_{r}\right|\right)}{\left|\mathbf{k}_{r}\right|^{2 \alpha+d-2}} \delta\left(\mathbf{k}_{r}+\mathbf{k}_{s}\right) d \mathbf{k}_{r} d \mathbf{k}_{s} .
\end{aligned}
$$

Using the change of variables made in the course of the proof of Proposition 4.3 we conclude therefore that

$$
\left|\left\langle\chi_{\lambda, p}^{(n)}(\mathcal{F}), F_{p}\right\rangle_{L^{2}}\right| \leq \frac{C}{\lambda^{e}}(1+|\log \lambda|)^{e+1}
$$

which yields (4.44) because $2 e+1=n$.

\section{A Multiple stochastic integration}

Suppose that $\mathbb{P}$ is a Gaussian, homogeneous, Borel measure over the space $\Omega$, introduced in Section 3 Denote by $\hat{\mathbf{F}}$ the corresponding Gaussian vector valued spectral measure on $\left(\mathbb{R}^{d}, \mathcal{B}\left(\mathbb{R}^{d}\right)\right)$. We suppose that the structure measure $\hat{\mathbf{R}}(d \mathbf{k})$ has the density $\mathbf{r}(\mathbf{k})=\left[r_{i j}(\mathbf{k})\right]$ w.r.t. the Lebesgue measure. Let $r(\mathbf{k}):=\operatorname{tr} \mathbf{r}(\mathbf{k}) \vee 1$. For a given integer $n \geq 1$ we consider the Borel measure on $\left(\mathbb{R}^{d}\right)^{2 n}$

$$
M_{2 n}\left(d \mathbf{k}_{1}, \ldots, d \mathbf{k}_{2 n}\right):=\sum_{\mathcal{F} \in \mathfrak{F}_{n}} \prod_{\widehat{p} q \in \mathcal{F}} \delta\left(\mathbf{k}_{p}+\mathbf{k}_{q}\right) \prod_{j=1}^{2 n} r\left(\mathbf{k}_{j}\right) d \mathbf{k}_{j}
$$

By $\mathcal{L}_{n}^{2}$ we denote the completion of the space of all complex valued, bounded Borel measurable functions $\psi:\left(\mathbb{R}^{d}\right)^{n} \rightarrow\left(\mathbb{C}^{d}\right)^{n}$ in the norm

$$
\|\psi\|_{\mathcal{L}_{n}^{2}}^{2}:=\int \underset{\left(\mathbb{R}^{d}\right)^{2 n}}{\ldots}\left(\left|\psi\left(\mathbf{k}_{1}, \ldots, \mathbf{k}_{n}\right)\right|^{2}+\left|\psi\left(\mathbf{k}_{n+1}, \ldots, \mathbf{k}_{2 n}\right)\right|^{2}\right) M_{2 n}\left(d \mathbf{k}_{1}, \ldots, d \mathbf{k}_{2 n}\right) .
$$

Suppose that $i \in \mathbb{Z}^{d}$ and $N \geq 1$. We define

$$
\square_{N}(i):=\left[\mathbf{k} \in \mathbb{R}^{d}: 2^{-N} i_{j} \leq k_{j}<2^{-N}\left(i_{j}+1\right), \forall j=1, \ldots, d\right] .
$$

For $\mathbf{i}:=\left(i_{1}, \ldots, i_{n}\right) \in\left(\mathbb{Z}^{d}\right)^{n}$ we let

$$
\square_{N}(\mathbf{i}):=\square_{N}\left(i_{1}\right) \times \ldots \times \square_{N}\left(i_{n}\right) .
$$

By $\mathcal{D}$ we denote the family of all such boxes. Its subfamily $\Pi$ is called an admissible dyadic partition of $\left(\mathbb{R}^{d}\right)^{2 n}$ if

(P1) $\bigcup_{\square \in \Pi} \square=\left(\mathbb{R}^{d}\right)^{2 n}$, 
(P2) for any two boxes $\square \neq \square^{\prime} \in \Pi$ we have $\square \cap \square^{\prime}=\emptyset$,

(P3) there exists $d_{0}>0$ such that $|\square| \geq d_{0}$ for all $\square \in \Pi$.

A set function $c: \Pi \rightarrow\left(\mathbb{C}^{d}\right)^{n}$ is called admissible if $c(\square)=0$ for all but finitely many boxes from an admissible $\Pi$. We denote by $\mathcal{A}$ the family of all admissible set functions.

For any multi-index $\mathbf{j}:=\left(j_{1}, \ldots, j_{n}\right) \in\{1, \ldots, d\}^{n}$ and $\square^{(N)}(\mathbf{i})$ given by (A.2) we let

$$
\hat{\mathbf{F}}_{\mathbf{j}}\left[\square_{N}(\mathbf{i})\right]=\prod_{p=1}^{n} \hat{F}_{j_{p}}\left[\square_{N}\left(i_{p}\right)\right] .
$$

Suppose that $c: \Pi \rightarrow\left(\mathbb{C}^{d}\right)^{n}$ is admissible. We define then $\psi(\mathbf{k}):=c(\square)$ for all $\mathbf{k} \in \square$. With some abuse of notation we call such a function admissible and denote by $\mathcal{A}_{n} \subset \mathcal{L}_{n}^{2}$ the space of all admissible functions. For any admissible function $\psi \in \mathcal{A}_{n}$ we define the $n$-tuple stochastic integral letting

$$
\mathcal{I}(\psi):=\sum_{\square \in \Pi} \sum_{\mathbf{j}} c_{\mathbf{j}}(\square) \cdot \hat{\mathbf{F}}_{\mathbf{j}}[\square]
$$

We shall also write $\int \ldots \int \psi\left(\mathbf{k}_{1}, \ldots, \mathbf{k}_{n}\right) \hat{\mathbf{F}}\left(d \mathbf{k}_{1}\right) \otimes \ldots \otimes \hat{\mathbf{F}}\left(d \mathbf{k}_{n}\right)$ to denote $\mathcal{I}(\psi)$. Below, we list some of the properties of $\mathcal{I}(\psi)$. They are elementary and their verification relies on the application of the definition so we leave this task to a reader.

Proposition A.1 (i) $\mathcal{A}_{n}$ is dense in $\mathcal{L}_{n}^{2}$ in the norm $\|\cdot\|_{\mathcal{L}_{n}^{2}}$.

(ii) The stochastic integral given by (A.4) is well defined, i.e. if there exist two admissible set functions $c_{1}, c_{2}$ corresponding to a given $\psi$ then the respective definitions of the stochastic integrals are identical.

(iii) We have $\mathcal{I}\left(a_{1} \psi_{1}+a_{2} \psi_{2}\right)=a_{1} \mathcal{I}\left(\psi_{1}\right)+a_{2} \mathcal{I}\left(\psi_{2}\right)$

(iv) Suppose that $\psi_{1}, \ldots, \psi_{n} \in \mathcal{A}_{1}$. Then $\psi_{1} \otimes \ldots \otimes \psi_{n} \in \mathcal{A}_{n}$ and

$$
\mathcal{I}\left(\psi_{1} \otimes \ldots \otimes \psi_{n}\right)=\prod_{j=1}^{n} \mathcal{I}\left(\psi_{j}\right) .
$$

(v) We have

$$
\begin{aligned}
\mathbb{E}\left[\mathcal{I}\left(\psi^{(1)}\right) \mathcal{I}^{*}\left(\psi^{(2)}\right)\right] & =\sum_{\mathcal{F} \in \mathfrak{F}_{n}} \sum_{j_{1}, \ldots, j_{2 n}=1}^{d} \int \ldots \int_{\left(\mathbb{R}^{d}\right)^{2 n}} \psi_{j_{1}, \ldots, j_{n}}^{(1)}\left(\mathbf{k}_{1}, \ldots, \mathbf{k}_{n}\right)\left(\psi_{j_{n+1}, \ldots, j_{2 n}}^{(2)}\right)^{*}\left(\mathbf{k}_{n+1}, \ldots, \mathbf{k}_{2 n}\right) \\
& \times \prod_{\hat{p} q \in \mathcal{F}} \hat{R}_{p q}\left(\mathbf{k}_{p}\right) \delta\left(\mathbf{k}_{p}-\mathbf{k}_{q}\right) \prod_{j=1}^{2 n} d \mathbf{k}_{j}
\end{aligned}
$$

As a direct consequence of property (v) and the definition of the norm on $\mathcal{L}_{n}$ we obtain.

Corollary A.2 $\mathcal{A}_{n} \ni \psi \mapsto \mathcal{I}(\psi)$ is a continuous functional that extends to the entire $\mathcal{L}_{n}^{2}$. The extension shall be called an n-tuple stochastic integral w.r.t. the spectral measure $\hat{\mathbf{F}}(d \mathbf{k})$. It has properties (iii)-(v) from Proposition A.1.

Proposition A.3 For any $\psi \in \mathcal{L}_{n}^{2}$ we let

$$
U^{\mathbf{x}} \psi\left(\mathbf{k}_{1}, \ldots, \mathbf{k}_{n}\right):=\exp \left\{i\left(\sum_{p=1}^{n} \mathbf{k}_{p}\right) \cdot \mathbf{x}\right\} \psi\left(\mathbf{k}_{1}, \ldots, \mathbf{k}_{n}\right) .
$$

Then, for any $\mathbf{x} \in \mathbb{R}^{d}$ we have $U^{\mathbf{x}} \psi \in \mathcal{L}_{n}^{2}$ and

$$
\mathcal{I}(\psi)\left(\tau_{\mathbf{x}} \omega\right)=\mathcal{I}\left(U^{\mathbf{x}} \psi\right)(\omega), \quad \mathbb{P}-\text { a.s. }
$$


Proof. We have

$$
\mathbf{F}\left(\tau_{\mathbf{x}+\mathbf{y}} \omega\right)=\int e^{i \mathbf{k} \cdot(\mathbf{x}+\mathbf{y})} \hat{\mathbf{F}}(d \mathbf{k} ; \omega)=\mathbf{F}\left(\tau_{\mathbf{x}}\left(\tau_{\mathbf{y}} \omega\right)\right)=\int e^{i \mathbf{k} \cdot \mathbf{x}} \hat{\mathbf{F}}\left(d \mathbf{k} ; \tau_{\mathbf{y}} \omega\right)
$$

for all $\mathbf{x}, \mathbf{y} \in \mathbb{R}^{d}$. Hence $\hat{\mathbf{F}}\left(d \mathbf{k} ; \tau_{\mathbf{y}} \omega\right)=e^{i \mathbf{k} \cdot \mathbf{y}} \hat{\mathbf{F}}(d \mathbf{k} ; \omega)$ and (A.6) follows.

Remark A different construction of a multiple stochastic integral can be obtained using the approach of Chapter 7.2 of [16]. Denote the stochastic integral, defined there, by

$$
\int \ldots \int^{(J)} \psi\left(\mathbf{k}_{1}, \ldots, \mathbf{k}_{n}\right) \cdot \hat{\mathbf{F}}\left(d \mathbf{k}_{1}\right) \otimes \ldots \otimes \hat{\mathbf{F}}\left(d \mathbf{k}_{n}\right) .
$$

Let $\mathcal{F}$ be a Feynman diagram labelled by $\{1, \ldots, n\}$. Let $n_{1}<\ldots<n_{a}$ be all the free vertices of $\mathcal{F}$ and let $\widehat{p q} \in E(\mathcal{F})$ denote the remaining $e:=(n-a) / 2$ edges. Set $T(\mathcal{F}) \psi:\left(\mathbb{R}^{d}\right)^{a} \rightarrow\left(\mathbb{C}^{d}\right)^{a}$

$$
T(\mathcal{F}) \psi_{i_{n_{1}} \ldots i_{n_{a}}}\left(\mathbf{k}_{n_{1}}, \ldots, \mathbf{k}_{n_{a}}\right):=\sum_{i_{p} i_{q}} \int \ldots \int \psi_{i_{1} \ldots i_{n}}\left(\mathbf{k}_{1}, \ldots, \mathbf{k}_{n}\right) \prod_{\widehat{p} q \in E(\mathcal{F})} r_{i_{p} i_{q}}\left(\mathbf{k}_{p}\right) \delta\left(\mathbf{k}_{p}+\mathbf{k}_{q}\right) d \mathbf{k}_{p} d \mathbf{k}_{q}
$$

for all $i_{n_{1}}, \ldots, i_{n_{a}}=1, \ldots, d$. Let

$$
I(\mathcal{F}):=\int \ldots \int^{(J)} T(\mathcal{F}) \psi\left(\mathbf{k}_{n_{1}}, \ldots, \mathbf{k}_{n_{l}}\right) \hat{\mathbf{F}}\left(d \mathbf{k}_{n_{1}}\right) \otimes \ldots \otimes \hat{\mathbf{F}}\left(d \mathbf{k}_{n_{l}}\right) .
$$

Using Theorem 7. 25 p. 99 and Corollary 3. 17 p. 28 of ibid. one can conclude that

$$
\int \ldots \int \psi\left(\mathbf{k}_{1}, \ldots, \mathbf{k}_{n}\right) \hat{\mathbf{F}}\left(d \mathbf{k}_{1}\right) \otimes \ldots \otimes \hat{\mathbf{F}}\left(d \mathbf{k}_{n}\right)=\sum_{\mathcal{F}} I(\mathcal{F})
$$

where the summation extends over all Feynman diagrams labelled by $\{1, \ldots, n\}$. We could use then equality (A.7) as the definition of the multiple stochastic integral.

\section{References}

[1] R. Adler, The Geometry of Random Fields, Wiley \& Sons, New York (1981).

[2] M. Avellaneda and A. Majda, Mathematical models with exact renormalization for turbulent transport, Comm. Math. Phys. 131, 1990, 381-429.

[3] M. Avellaneda and A. Majda, Mathematical models with exact renormalization for turbulent transport II. Fractal interfaces, non-Gaussian statistics and the sweeping effect. Comm. Math. Phys. 146, 1992, 139-204.

[4] M. Avellaneda and A. Majda, An integral representation and bounds on the effective diffusivity in passive advection by laminar and turbulent flows. Comm. Math. Phys. 138, 1991, 339-391.

[5] M. Avellaneda and A. Majda, Superdiffusion in nearly stratified flows. J. Stat. Phys. 69, 1992, 689-729.

[6] Yu. Belyaev, Analytic Random Processes, Teor. Prob. Appl., 4, 1959, 437-444.

[7] G. Ben Arous and H. Owhadi, Super-diffusivity in a shear flow model from perpetual homogenization. Comm. Math. Phys. 227, 2002, 281-302. 
[8] C. Bernardin, Fluctuations in the occuption time of a site in an asymetric simple exclusion process, Ann. of Prob., 32, 2003, 855-879.

[9] M. Chertkov and G. Falkovich, Anomalous scaling exponents of white-advected passive scalar, Phys. Rev. Lett., 76, 1996, 2706-2709.

[10] M. Chertkov, G. Falkovich, I. Kolokolov and V. Lebedev, Normal and anomalous scaling of the fourth-order correlation function of a randomly advected passive scalar Phys. Rev. E, 52, 1995, 4924-4941.

[11] A. Fannjiang and T. Komorowski, Fractional Brownian motions in a limit of turbulent transport, Ann. Appl. Probab. 10, 2000, 1100-1120.

[12] A. Fannjiang, T. Komorowski and Sz. Peszat, Lagrangian dynamics for a passive tracer in a class of Gaussian Markovian flows. Stoch. Proc. Appl. 97, 2002, 171-198.

[13] G.M. Fichtenholz, Course in differential and integral calculus. (In Russian) FizMatGIz, Moscow, 1959.

[14] K. Gawedzki and A. Kupiainen, Anomalous scaling of the passive scalar, Phys. Rev. Lett. 75, $1995,3834-3837$.

[15] H. Hochstadt, The Functions of Mathematical Physics, Dover, New York, 1986.

[16] S. Janson, Gaussian Hilbert Spaces, Cambridge Tracts in Math. 129, Cambridge Univ. Press, 1997.

[17] H. Kesten, G. C. Papanicolaou, A limit theorem for turbulent diffusion, Comm. Math. Phys. 65, 1979, 97-128.

[18] T. Komorowski and S. Olla, On the superdiffusive behavior of passive tracer with a Gaussian drift, Jour. Stat. Phys. 108, 2002, 647-668.

[19] T. Komorowski and S. Olla, On the sector condition and homogenization of diffusions with a Gaussian drift, Jour. Funct. Anal. 197, 2003, 179-211.

[20] R. Kraichnan, Anomalous scaling of a randomly advected passive scalar, Phys. Rev. Lett. 72, 1994, 1016-1019.

[21] A. J. Majda and P. R. Kramer, Simplified models for turbulent diffusion: Theory, numerical modelling and physical phenomena, Physics Reports, 314, 1999, 237-574.

[22] H. Owhadi, Averaging versus chaos in turbulent transport? Comm. Math. Phys. 247, 2004, $553-599$.

[23] Yu. A. Rozanov, Stationary Random Processes, Holden-Day, San Fransisco, Cambridge, London, Amsterdam 1967.

[24] G. Samorodnitsky and M. S. Taqqu, Stable Non-Gaussian Random Processes: Stochastic Models with Infinite Variance, Chapman \& Hall, New York, 1994.

[25] B. Shraiman and E. Siggia, Anomalous scaling and small scale anisotropy of a passive scalar in turbulent flow, C.R. Acad. Sci., Paris, 321, 1995, 279-284.

[26] S. Wunsch, Simple passive scalar advection-diffusion model, Phys. Rev. E, 58, 1998, 5757-5764. 